\title{
A theorem in the geometry of numbers
}

\author{
By \\ Moto-o TAKaHASHI
}

Let $M$ be the exterior of a knot in the 3-sphere $S^{3}$ (or more generally a compact 3-manifold with a torus as boundary) and let $M(p, r)$ be the closed 3manifold obtained from $M$ by $(p, r)$-Dehn surgery. ( $p, r$ are co-prime integers.) Roughly speaking, the number of non-trivial representations of the fundamental group of $M(p, r)$ to PSL $(2, C)$ is given by the formula

$$
\sum_{i=1}^{n}\left|\alpha_{i} p-\beta_{i} r\right|-e
$$

So, if this number is positive, then $M(p, r)$ is not simply-connected. So, the calculation of this number is useful for studying Poincaré conjecture.

In this paper we shall prove a theorem about the functions of the above form, purely in the geometry of numbers, independent of the topology of 3manifolds. We use Minkowski's theorem in proving this theorem. Moreover we introduce the notion of $\mathrm{C}$-system as a tool for proving the theorem. In future we wish to apply this theorem to the study of Poincaré conjecture.

Let $L^{\prime}=\boldsymbol{Z} \times \boldsymbol{Z}-\{(0,0)\}$

TheOREM 1. Let $\alpha_{i}, \beta_{i}(i=1, \ldots, m), \gamma_{j}, \delta_{j}(j=1, \ldots, n), e, f$ be real numbers such that $\alpha_{i} \beta_{k}-\beta_{i} \alpha_{k} \neq 0 \quad(i \neq k), \quad \gamma_{j} \delta_{\ell}-\delta_{j} \gamma_{\ell} \neq 0(j \neq l), \quad e>0, f>0$. Suppose that, for all $(x, y) \in L^{\prime}$,

$$
\sum_{i=1}^{m}\left|\alpha_{i} x-\beta_{i} y\right| \geq e
$$

and

$$
\sum_{j=1}^{n}\left|\gamma_{j} x-\delta_{j} y\right| \geq f
$$

Then, 


$$
\sum_{i=1}^{m} \sum_{j=1}^{n}\left|\alpha_{i} \delta_{j}-\beta_{i} \gamma_{j}\right| \geq e f
$$

Moreover, the equlity in the last inequality holds only when $m=n=2$ and $\left|\alpha_{1} \beta_{2}-\beta_{1} \alpha_{2}\right|=e^{2} / 2,\left|\gamma_{1} \delta_{2}-\delta_{1} \gamma_{2}\right|=f^{2} / 2$.

Corollary 2. Let $\alpha_{i}, \beta_{i}(i=1, \ldots, m), \gamma_{j}, \delta_{j}(j=1, \ldots, n), e, f$ be as in the Theorem 1. Then, for all $(u, v) \in L^{\prime}$,

$$
\sum_{i=1}^{m} \sum_{j=1}^{n}\left|\alpha_{i} \gamma_{j} u-\left(\alpha_{i} \delta_{j}+\beta_{i} \gamma_{j}\right) v\right| \geq e f
$$

Moreover, the equality in the last inequality holds only when either

(i) $u= \pm 1, v=0$, or (ii) $m=n=2$.

We first prove that Theorem 1 implies Corollary 2. Let $(u, v) \in L^{\prime}$.

Case 1. $v=0$.

Then, $u \neq 0$ and, since $u$ is an integer, $|u| \geq 1$. According to the hypothesis (1). (2) of the Corollary 2 with $(x, y)=(1,0)$, we have

$$
\sum_{i=1}^{m}\left|\alpha_{i}\right| \geq e \quad \text { and } \quad \sum_{j=1}^{n}\left|\gamma_{j}\right| \geq f
$$

Therefore,

$$
\sum_{i=1}^{m} \sum_{j=1}^{n}\left|\alpha_{i} \gamma_{j}\right| \geq e f
$$

So,

$$
\sum_{i=1}^{m} \sum_{j=1}^{n}\left|\alpha_{i} \gamma_{j} u-\left(\alpha_{i} \delta_{j}+\beta_{i} \gamma_{j}\right) v\right|=\sum_{i=1}^{m} \sum_{j=1}^{n}\left|\alpha_{i} \gamma_{j}\right||u| \geq \sum_{i=1}^{m} \sum_{j=1}^{n}\left|\alpha_{i} \gamma_{j}\right| \geq e f .
$$

Moreover, if $|u|>1$, then

$$
\sum_{i=1}^{m} \sum_{j=1}^{n}\left|\alpha_{i} \gamma_{j} u-\left(\alpha_{i} \delta_{j}+\beta_{i} \gamma_{j}\right) v\right|>e f
$$

Case 2. $v \neq 0$.

Let $(x, y) \in L^{\prime}$. Then, $(u y-v x, v y) \in L^{\prime}$. Then, by the hypothesis (2), we have

$$
\sum_{j=1}^{n}\left|\gamma_{j}(u y-v x)-\delta_{j}(v y)\right| \geq f
$$


that is,

$$
\sum_{j=1}^{n}\left|\left(\gamma_{j} v\right) x-\left(\gamma_{j} u-\delta_{j} v\right) y\right| \geq f
$$

for all $(x, y) \in L^{\prime}$. Moreover, if $j \neq \ell$,

$$
\left(\gamma_{j} v\right)\left(\gamma_{\ell} u-\delta_{\ell} v\right)-\left(\gamma_{j} u-\delta_{j} v\right)\left(\gamma_{\ell} v\right)=-\left(\gamma_{j} \delta_{\ell}-\delta_{j} \gamma_{\ell}\right) v^{2} \neq 0 .
$$

So, we can use Theorem 1 for (1) and (3) (that is, we take $\gamma_{j} v$ and $\gamma_{j} u-\delta_{j} v$ instead of $\gamma_{j}$ and $\delta_{j}$, respectively).

Then, we have

$$
\sum_{i=1}^{m} \sum_{j=1}^{n}\left|\alpha_{i}\left(\gamma_{j} u-\delta_{j} v\right)-\beta_{i}\left(\gamma_{j} v\right)\right| \geq e f
$$

that is,

$$
\sum_{i=1}^{m} \sum_{j=1}^{n}\left|\alpha_{i} \gamma_{j} u-\left(\alpha_{i} \delta_{j}+\beta_{i} \gamma_{j}\right) v\right| \geq e f
$$

as was to be proved. Moreover, if the equality holds in the last inequality, then by Theorem 1, we have $m=n=2$

In order to prove Theorem 1, we can assume that $e=f=1$ without loss of generality. First prove the following lemma, which is a special case of Theorem 1 .

Lemma 3. Let $\alpha_{i}, \beta_{i}(i=1, \ldots, m)$ be real numbers such that $\alpha_{i} \beta_{k}-\beta_{i} \alpha_{k} \neq 0$ $(i \neq k)$. Suppose that, for all $(x, y) \in L^{\prime}$,

$$
\sum_{i=1}^{m}\left|\alpha_{i} x-\beta_{i} y\right| \geq 1
$$

Then,

$$
\sum_{i=1}^{m} \sum_{j=1}^{m}\left|\alpha_{i} \beta_{j}-\beta_{i} \alpha_{j}\right|\left(=2 \sum_{i<j}\left|\alpha_{i} \beta_{j}-\beta_{i} \alpha_{j}\right|\right) \geq 1
$$

Moreover, the equality holds in the last inequality only when $m=2$ and $\left|\alpha_{1} \beta_{2}-\beta_{1} \alpha_{2}\right|=\frac{1}{2}$.

Proof of Lemma 3. Case 1. $m=1$.

Clearly, the hypothesis of the lemma never holds. 
Case 2. $m=2$.

Then, the hypotheses are:

$$
\alpha_{1} \beta_{2}-\beta_{1} \alpha_{2} \neq 0
$$

and

$$
\left|\alpha_{1} x-\beta_{1} y\right|+\left|\alpha_{2} x-\beta_{2} y\right| \geq 1,
$$

for all $(x, y) \in L^{\prime}$. The conclusion of the theorem becomes

$$
2\left|\alpha_{1} \beta_{2}-\beta_{1} \alpha_{2}\right| \geq 1
$$

Now, the domain

$$
\Delta=\left\{(x, y) \in \boldsymbol{R}^{2}|\cdot| \alpha_{1} x-\beta_{1} y|+| \alpha_{2} x-\beta_{2} y \mid<1\right\}
$$

is the interior of a parallelogram which is symmetric with respect to the origin, and the vertices of which are

$$
\pm\left(\beta_{1} /\left|\alpha_{1} \beta_{2}-\beta_{1} \alpha_{2}\right|, \alpha_{1} /\left|\alpha_{1} \beta_{2}-\beta_{1} \alpha_{2}\right|\right)
$$

and

$$
\pm\left(\beta_{2} /\left|\alpha_{1} \beta_{2}-\beta_{1} \alpha_{2}\right|, \alpha_{2} /\left|\alpha_{1} \beta_{2}-\beta_{1} \alpha_{2}\right|\right)
$$

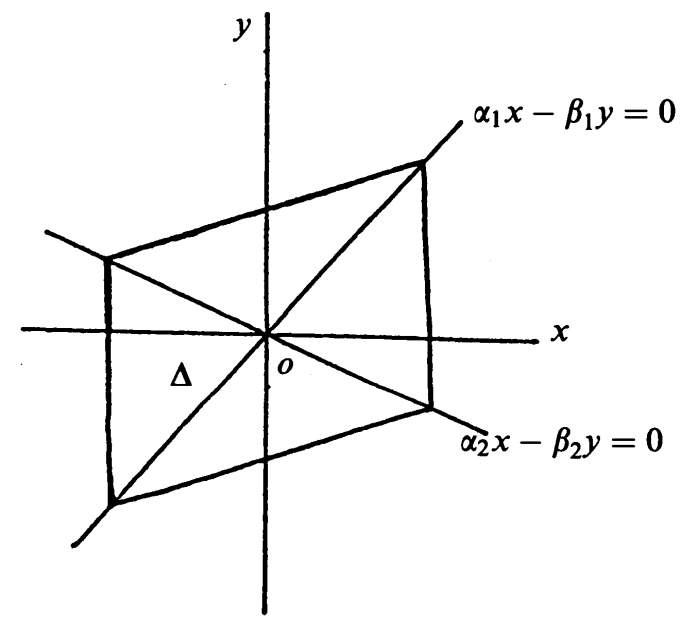

Figure 1

So, the area of $\Delta$ is $2 / \alpha_{1} \beta_{2}-\beta_{1} \alpha_{2} \mid$. By the hypothesis of the lemma, $\Delta$ does not contain elements of $L^{\prime}$. So, by Minkowski's theorem, the area of $\Delta$ must be less than or equal to 4 . Thus, $2 /\left|\alpha_{1} \beta_{2}-\beta_{1} \alpha_{2}\right| \leq 4$, that is, $2\left|\alpha_{1} \beta_{2}-\beta_{1} \alpha_{2}\right| \geq 1$, as was to be proved. If the equality holds, then $\left|\alpha_{1} \beta_{2}-\beta_{1} \alpha_{2}\right|=1 / 2$. 
Case 3. $m>2$.

We shall prove that

$$
\sum_{i=1}^{m} \sum_{j=1}^{m}\left|\alpha_{i} \beta_{j}-\beta_{i} \alpha_{j}\right|>1
$$

Consider the domain

$$
\Delta=\left\{(x, y) \in \boldsymbol{R}^{2}\left|\sum_{i=1}^{m}\right| \alpha_{i} x-\beta_{i} y \mid<1\right\}
$$

$\Delta$ is the interior of a convex $2 m$-gon $D$ which is symmetric with respect to the origin. Let $P_{1}, P_{2}, \ldots, P_{2 m}$ be the vertices of $D$ (we assume that these are ordered counterclockwise). By changing the subscripts of $\alpha_{i}, \beta_{i}$, if necessary, we can assume without loss of generality that $P_{i}$ and $P_{i+m}$ are on the line $\alpha_{i} x-\beta_{i} y=0$ $(i=1, \ldots, m)$. Let $\left(a_{i}, b_{i}\right)$ be the coordinate of $P_{i}$. It holds that $a_{i+m}=-a_{i}$, $b_{i+m}=-b_{i}$.

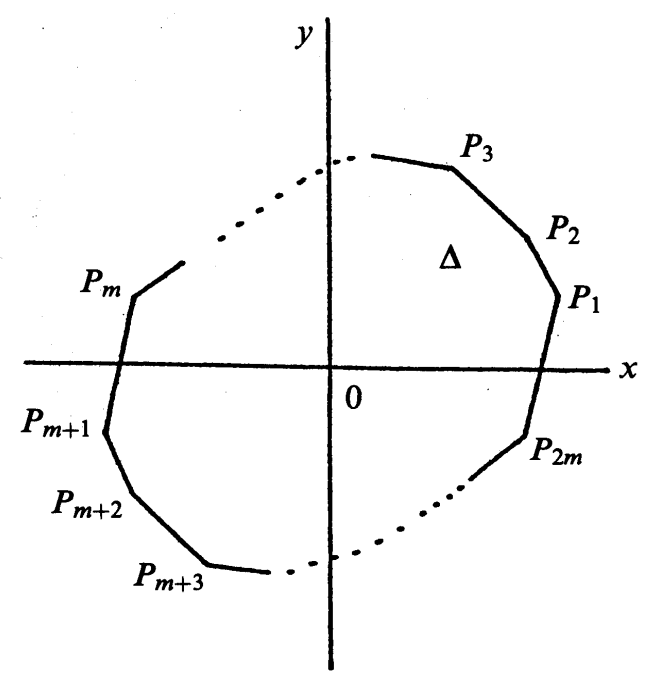

Figure 2

Since $P_{i}$ is on the line $\alpha_{i} x-\beta_{i} y=0$, there is a $\lambda_{i} \neq 0$ such that $\alpha_{i}=\lambda_{i} b_{i}$ and $\beta_{i}=\lambda_{i} a_{i}$. It holds that $\lambda_{i+m}=-\lambda_{i}(i=1, \ldots, m)$. Let $u_{i}=\left|\lambda_{i}\right|=\left|\lambda_{i+m}\right|$. Since $P_{i}=\left(a_{i}, b_{i}\right)$ is on $D$, we have

$$
\sum_{k=1}^{m}\left|\alpha_{k} a_{i}-\beta_{k} b_{i}\right|=1
$$


that is,

$$
\sum_{k=1}^{m} \mu_{k}\left|b_{k} a_{i}-a_{k} b_{i}\right|=1
$$

So, if we put $c(k, i)=\left|b_{k} a_{i}-a_{k} b_{i}\right|$, we have

$$
\sum_{k=1}^{m} \mu_{k} c(k, i)=1 \quad(i=1, \ldots, m)
$$

$\{c(k, i): 1 \leq k, i \leq m\}$ satisfies the following.

C1. (i) $c(k, k)=0$,

(ii) $c(k, i)>0 \quad(k \neq i)$,

C2. $c(k, i)=c(i, k)$,

C3. for $1 \leq i<j<k<\ell \leq m$,

$$
c(i, k) c(j, \ell)=c(i, j) c(k, \ell)+c(i, \ell) c(j, k) .
$$

C4. (i) $c(m, 1)+c(1,2)>c(m, 2)$,

(ii) for $2 \leq i \leq m-1$,

$$
c(i-1, i)+c(i, i+1)>c(i-1, i+1),
$$

(iii) $c(m-1, m)+c(m, 1)>c(m-1,1)$.

$\mathrm{C} 1$ and $\mathrm{C} 2$ are obvious. We prove C3. From the Figure 2, we have $a_{i} b_{j}$ $b_{i} a_{j}>0$, for $1 \leq i<j \leq m$. Hence, $c(i, j)=a_{i} b_{j}-b_{i} a_{j}$. Similarly, if $1 \leq i<j<$ $k<\ell \leq m$, then

$$
\begin{gathered}
c(i, k)=a_{i} b_{k}-b_{i} a_{k}, \quad c(j, \ell)=a_{j} b_{\ell}-b_{j} a_{\ell}, \quad c(k, \ell)=a_{k} b_{\ell}-b_{k} a_{\ell} \\
c(i, \ell)=a_{i} b_{\ell}-b_{i} a_{\ell}, \quad c(j, k)=a_{j} b_{k}-b_{j} a_{k} .
\end{gathered}
$$

Thus, C3 holds.

Next we prove C4 (ii). C4 (i) and C4 (iii) can be proved similarly. Now, by (4) above,

$$
1=\sum_{\ell=1}^{m} \mu_{\ell} c(\ell, i+1)=\sum_{\ell=1}^{m} \mu_{\ell} c(\ell, i-1)=\sum_{\ell=1}^{m} \mu_{\ell} c(\ell, i) .
$$


So,

$$
\begin{aligned}
c(i- & 1, i)+c(i, i+1)-c(i-1, i+1) \\
= & \left\{\sum_{\ell=1}^{m} \mu_{\ell} c(\ell, i+1)\right\} c(i-1, i)+\left\{\sum_{\ell=1}^{m} \mu_{\ell} c(\ell, i-1)\right\} c(i, i+1) \\
& -\left\{\sum_{\ell=1}^{m} \mu_{\ell} c(\ell, i)\right\} c(i-1, i+1) \\
= & \sum_{\ell=1}^{m} \mu_{\ell}\{c(\ell, i+1) c(i-1, i)+c(\ell, i-1) c(i, i+1)-c(\ell, i) c(i-1, i+1)\} .
\end{aligned}
$$

If $\ell<i-1$ or $i+1<\ell$, then by $\mathrm{C} 3$,

$$
c(\ell, i+1) c(i-1, i)+c(\ell, i-1) c(i, i+1)-c(\ell, i) c(i-1, i+1)=0 .
$$

Also, if $\ell=i-1$, then by $\mathrm{C} 1$ and $\mathrm{C} 2$,

$$
\begin{aligned}
& c(\ell, i+1) c(i-1, i)+c(\ell, i-1) c(i, i+1)-c(\ell, i) c(i-1, i+1) \\
& \quad=c(i-1, i+1) c(i-1, i)+c(i-1, i-1) c(i, i+1)-c(i-1, i) c(i-1, i+1)=0 .
\end{aligned}
$$

Similarly, if $\ell=i+1$, then

$$
\begin{aligned}
& c(\ell, i+1) c(i-1, i)+c(\ell, i-1) c(i, i+1)-c(\ell, i) c(i-1, i+1) \\
& \quad=c(i+1, i+1) c(i-1, i)+c(i+1, i-1) c(i, i+1)-c(i+1, i) c(i-1, i+1)=0 .
\end{aligned}
$$

If $\ell=i$, then

$$
\begin{aligned}
& c(\ell, i+1) c(i-1, i)+c(\ell, i-1) c(i, i+1)-c(\ell, i) c(i-1, i+1) \\
& \quad=2 c(i-1, i) c(i, i+1)
\end{aligned}
$$

Hence

$$
\begin{aligned}
c(i & -1, i)+c(i, i+1)-c(i-1, i+1) \\
& =\sum_{\ell=1}^{m} \mu_{\ell}\{c(\ell, i+1) c(i-1, i)+c(\ell, i-1) c(i, i+1)-c(\ell, i) c(i-1, i+1)\} \\
& =\mu_{i}\{2 c(i-1, i) c(i, i+1)\}>0 .
\end{aligned}
$$

Hence

$$
c(i-1, i)+c(i, i+1)>c(i-1, i+1) .
$$


From (*) follows that

M1. $\mu_{1}=\frac{1}{2 c(m, 1)}+\frac{1}{2 c(1,2)}-\frac{c(m, 2)}{2 c(m, 1) c(1,2)}$,

M2. For $2 \leq i \leq m-1$,

$$
\mu_{i}=\frac{1}{2 c(i-1, i)}+\frac{1}{2 c(i, i+1)}-\frac{c(i-1, i+1)}{2 c(i-1, i) c(i, i+1)}
$$

M3. $\mu_{m}=\frac{1}{2 c(m-1, m)}+\frac{1}{2 c(m, 1)}-\frac{c(m-1,1)}{2 c(m-1, m) c(m, 1)}$.

We must show

$$
\sum_{i=1}^{m} \sum_{j=1}^{m}\left|\alpha_{i} \beta_{j}-\beta_{i} \alpha_{j}\right|>1
$$

Now,

$$
\begin{aligned}
\sum_{i=1}^{m} \sum_{j=1}^{m}\left|\alpha_{i} \beta_{j}-\beta_{i} \alpha_{j}\right|= & \sum_{i=1}^{m} \sum_{j=1}^{m} \mu_{i} \mu_{j} c(i, j) \\
= & \sum_{i=1}^{m} \mu_{i}\left\{\sum_{j=1}^{m} \mu_{j} c(i, j)\right\}=\sum_{i=1}^{m} \mu_{i} \\
= & \left\{\frac{1}{2 c(m, 1)}+\frac{1}{2 c(1,2)}-\frac{c(m, 2)}{2 c(m, 1) c(1,2)}\right\} \\
& +\sum_{i=2}^{m-1}\left\{\frac{1}{2 c(i-1, i)}+\frac{1}{2 c(i, i+1)}-\frac{c(i-1, i+1)}{2 c(i-1, i) c(i, i+1)}\right\} \\
& +\left\{\frac{1}{2 c(m-1, m)}+\frac{1}{2 c(m, 1)}-\frac{c(m-1,1)}{2 c(m-1, m) c(m, 1)}\right\} \\
= & {\left[\left\{\sum_{i=1}^{m-1} \frac{1}{c(i, i+1)}+\frac{1}{c(m, 1)}\right\}-\left\{\frac{c(m, 2)}{2 c(m, 1) c(1,2)}\right.\right.} \\
& \left.\left.+\sum_{i=2}^{m-1} \frac{c(i-1, i+1)}{2 c(i-1, i) c(i, i+1)}+\frac{c(m-1,1)}{2 c(m-1, m) c(m, 1)}\right\}\right]
\end{aligned}
$$

Here we have used (4) and M1, M2, M3. So, we have to show 


$$
\begin{aligned}
& \left\{\sum_{i=1}^{m-1} \frac{1}{c(i, i+1)}+\frac{1}{c(m, 1)}\right\}-\left\{\frac{c(m, 2)}{2 c(m, 1) c(1,2)}\right. \\
& \left.\quad+\sum_{i=2}^{m-1} \frac{c(i-1, i+1)}{2 c(i-1, i) c(i, i+1)}+\frac{c(m-1,1)}{2 c(m-1, m) c(m, 1)}\right\}>1 .
\end{aligned}
$$

Now, the area of the domain $\Delta$ is

$$
\begin{aligned}
\sum_{i=1}^{m}\left|a_{i} b_{i+1}-b_{i} a_{i+1}\right| & =\sum_{i=1}^{m-1}\left|a_{i} b_{i+1}-b_{i} a_{i+1}\right|+\left|a_{m} b_{1}-b_{m} a_{1}\right| \\
& =\sum_{i=1}^{m-1} c(i, i+1)+c(m, 1) .
\end{aligned}
$$

By the assumption of the lemma, $\Delta$ does not contain elements of $L^{\prime}$. So by Minkowski's theorem, the area of $\Delta$ is less than or equal to 4 . That is,

$$
\sum_{i=1}^{m-1} c(i, i+1)+c(m, 1) \leq 4
$$

So, in order to prove (5), it suffices to show that

$$
\begin{aligned}
& \left\{\sum_{i=1}^{m-1} c(i, i+1)+c(m, 1)\right\}\left[\left\{\sum_{i=1}^{m-1} \frac{1}{c(i, i+1)}+\frac{1}{c(m, 1)}\right\}\right. \\
& \left.\quad-\left\{\frac{c(m, 2)}{2 c(m, 1) c(1,2)}+\sum_{i=2}^{m-1} \frac{c(i-1, i+1)}{2 c(i-1, i) c(i, i+1)}+\frac{c(m-1,1)}{2 c(m-1, m) c(m, 1)}\right\}\right]>4 .
\end{aligned}
$$

DEFinition. A system $C=\langle c(i, j): i, j=1, \ldots, m\rangle$ of real numbers $(m \geq 3)$ is called a $C$-system if it satisfies the above C1-C4. We set $\mu(C)=m$.

If $C$ satisfies $\mathrm{C} 1-\mathrm{C} 3$ and

$\mathrm{C} 4^{\prime}$. (i) $c(m, 1)+c(1,2) \geq c(m, 2)$,

(ii) for $2 \leq i \leq m-1$,

$$
c(i-1, i)+c(i, i+1) \geq c(i-1, i+1),
$$

(iii) $c(m-1, m)+c(m, 1) \geq c(m-1,1)$,

instead of $\mathrm{C} 4$, then $C$ is called a semi- $C$-system. Also, we set $\mu(C)=m$.

In order to prove Lemma 3 , it suffices to show the following. 
LEMMA 4. (i) For every C-system C, (6) holds, i.e.

$$
\begin{gathered}
\left\{\sum_{i=1}^{m-1} c(i, i+1)+c(m, 1)\right\}\left\{\sum_{i=1}^{m-1} \frac{1}{c(i, i+1)}+\frac{1}{c(m, 1)}-\frac{c(m, 2)}{2 c(m, 1) c(1,2)}\right. \\
\left.-\sum_{i=2}^{m-1} \frac{c(i-1, i+1)}{2 c(i-1, i) c(i, i+1)}-\frac{c(m-1,1)}{2 c(m-1, m) c(m, 1)}\right\}>4 .
\end{gathered}
$$

(ii) For every semi-C-system $C$, the following (7) holds.

$$
\begin{gathered}
\left\{\sum_{i=1}^{m-1} c(i, i+1)+c(m, 1)\right\}\left\{\sum_{i=1}^{m-1} \frac{1}{c(i, i+1)}+\frac{1}{c(m, 1)}-\frac{c(m, 2)}{2 c(m, 1) c(1,2)}\right. \\
\left.-\sum_{i=2}^{m-1} \frac{c(i-1, i+1)}{2 c(i-1, i) c(i, i+1)}-\frac{c(m-1,1)}{2 c(m-1, m) c(m, 1)}\right\} \geq 4 .
\end{gathered}
$$

Proof. We prove Lemma 4 by the induction on $m=\mu(C)$.

Case 1. $m=3$.

Then,

$$
\begin{aligned}
L= & \{c(1,2)+c(2,3)+c(3,1)\}\left\{\frac{1}{c(1,2)}+\frac{1}{c(2,3)}+\frac{1}{c(3,1)}-\frac{c(3,2)}{2 c(3,1) c(1,2)}\right. \\
& \left.-\frac{c(1,3)}{2 c(1,2) c(2,3)}-\frac{c(2,1)}{2 c(2,3) c(3,1)}\right\}-4 \\
= & \frac{\{c(1,2)+c(2,3)-c(3,1)\}\{c(2,3)+c(3,1)-c(1,2)\}\{c(3,1)+c(1,2)-c(2,3)\}}{2 c(1,2) c(2,3) c(3,1)}
\end{aligned}
$$

Hence if $C$ is a $C$-system, then $L>0$, and if $C$ is a semi- $C$-system, then $L \geq 0$. Case 2. $m \geq 4$.

We assume that (6) holds for every $C$-system $C$ with $\mu(C)<m$ and that (7) holds for every semi- $C$-system $C$ with $\mu(C)<m$. We shall show that (6) holds for every $C$-system $C$ with $\mu(C)=m$ and that (7) holds for every semi- $C$-system $C$ with $\mu(C)=m$. (From now on to the end of the proof of Lemma 4, we fix $m$ but $C$ varies.)

Under the above hypothesis, we first prove the following.

Proposition 5. Let $C$ be a semi-C-system with $\mu(C)=m$. Suppose that one of the following holds. 
(i) $c(m, 1)+c(1,2)=c(m, 2)$,

(ii) $c(k-1, k)+c(k, k+1)=c(k-1, k+1)$, for some $k(2 \leq k \leq m-1)$,

(iii) $c(m-1, m)+c(m, 1)=c(m-1,1)$.

Then, (7) holds for $C$.

Proof of Proposition 5. By a cyclic change of subscripts we can assume that

(iii) $c(m-1, m)+c(m, 1)=c(m-1,1)$.

Let $C^{\prime}=\langle c(i, j): i, j=1, \ldots, m-1\rangle . C^{\prime}$ satisfies $\mathrm{C} 1-\mathrm{C} 3$. Also, it satisfies

$$
c(i-1,1)+c(i, i+1) \geq c(i-1, i+1)
$$

for $2 \leq i \leq m-2$. Also,

$$
\begin{aligned}
& \{c(m-1,1)+c(1,2)-c(m-1,2)\} c(1, m) \\
& \quad=c(m-1,1) c(1, m)+c(1,2) c(1, m)-c(m-1,2) c(1, m) \\
& \quad=c(m-1,1) c(1, m)+c(1,2) c(1, m)-c(m-1,1) c(2, m)+c(1,2) c(m-1, m) \\
& \quad=c(m-1,1) c(1, m)-c(m-1,1) c(2, m)+c(1,2)\{c(1, m)+c(m-1, m)\} \\
& \quad=c(m-1,1) c(1, m)-c(m-1,1) c(2, m)+c(1,2) c(m-1,1) \\
& \quad=c(m-1,1)\{c(1, m)+c(1,2)-c(2, m)\} \geq 0 .
\end{aligned}
$$

Hence

$$
c(m-1,1)+c(1,2) \geq c(m-1,2)
$$

Also,

$$
\begin{aligned}
\{c( & -2, m-1)+c(m-1,1)-c(m-2,1)\} c(m-1, m) \\
= & c(m-2, m-1) c(m-1, m)+c(m-1,1) c(m-1, m)-c(m-2,1) c(m-1, m) \\
= & c(m-2, m-1) c(m-1, m)+c(m-1,1) c(m-1, m)-c(m-1,1) c(m-2, m) \\
& \quad+c(1, m) c(m-2, m-1) \\
= & c(m-2, m-1)\{c(m-1, m)+c(m, 1)\}+c(m-1,1) c(m-1, m) \\
& \quad-c(m-1,1) c(m-2, m)
\end{aligned}
$$




$$
\begin{aligned}
& =c(m-2, m-1) c(m-1,1)+c(m-1,1) c(m-1, m)-c(m-1,1) c(m-2, m) \\
& =c(m-1,1)\{c(m-2, m-1)+c(m-1, m)-c(m-2, m)\} \geq 0 .
\end{aligned}
$$

Hence

$$
c(m-2, m-1)+c(m-1,1) \geq c(m-2,1) .
$$

Thus, $C^{\prime}$ satisfies $C 4^{\prime}$. Hence $C^{\prime}$ is a semi- $C$-system with $\mu\left(C^{\prime}\right)=m-1$. Hence (7) holds for $C^{\prime}$ by the assumption. So,

$$
\begin{aligned}
J= & \left\{\sum_{i=1}^{m-2} c(i, i+1)+c(m-1,1)\right\}\left\{\sum_{i=1}^{m-2} \frac{1}{c(i, i+1)}+\frac{1}{c(m-1,1)}\right. \\
& -\frac{c(m-1,2)}{2 c(m-1,1) c(1,2)}-\sum_{i=2}^{m-2} \frac{c(i-1, i+1)}{2 c(i-1, i) c(i, i+1)} \\
& \left.-\frac{c(m-2,1)}{2 c(m-2, m-1) c(m-1,1)}\right\} \geq 4 .
\end{aligned}
$$

We must show that

$$
\begin{aligned}
K= & \left\{\sum_{i=1}^{m-1} c(i, i+1)+c(m, 1)\right\}\left\{\sum_{i=1}^{m-1} \frac{1}{c(i, i+1)}+\frac{1}{c(m, 1)}-\frac{c(m, 2)}{2 c(m, 1) c(1,2)}\right. \\
& \left.-\sum_{i=2}^{m-1} \frac{c(i-1, i+1)}{2 c(i-1, i) c(i, i+1)}-\frac{c(m-1,1)}{2 c(m-1, m) c(m, 1)}\right\} \geq 4 .
\end{aligned}
$$

It suffices to show that $J=K$. Now,

$$
\begin{aligned}
\sum_{i=1}^{m-2} c(i, i+1)+c(m-1,1) & =\sum_{i=1}^{m-2} c(i, i+1)+c(m-1, m)+c(m, 1) \\
& =\sum_{i=1}^{m-1} c(i, i+1)+c(m, 1) .
\end{aligned}
$$

Also, 


$$
\begin{aligned}
& {\left[\sum_{i=1}^{m-2} \frac{1}{c(i, i+1)}+\frac{1}{c(m-1,1)}-\frac{c(m-1,2)}{2 c(m-1,1) c(1,2)}-\sum_{i=2}^{m-2} \frac{c(i-1, i+1)}{2 c(i-1, i) c(i, i+1)}\right.} \\
& \left.-\frac{c(m-2,1)}{2 c(m-2, m-1) c(m-1,1)}\right]-\left[\sum_{i=1}^{m-1} \frac{1}{c(i, i+1)}+\frac{1}{c(m, 1)}-\frac{c(m, 2)}{2 c(m, 1) c(1,2)}\right. \\
& \left.-\sum_{i=2}^{m-1} \frac{c(i-1, i+1)}{2 c(i-1, i) c(i, i+1)}-\frac{c(m-1,1)}{2 c(m-1, m) c(m, 1)}\right] \\
& =-\frac{1}{c(m-1, m)}+\frac{1}{c(m-1,1)}-\frac{1}{c(m, 1)}-\frac{c(m-1,2)}{2 c(m-1,1) c(1,2)}+\frac{c(m, 2)}{2 c(m, 1) c(1,2)} \\
& +\frac{c(m-2, m)}{2 c(m-2, m-1) c(m-1, m)}-\frac{c(m-2,1)}{2 c(m-2, m-1) c(m-1,1)} \\
& +\frac{c(m-1,1)}{2 c(m-1, m) c(m, 1)} \\
& =-\frac{1}{c(m-1, m)}+\frac{1}{c(m-1,1)}-\frac{1}{c(m, 1)}+\frac{-c(m-1,2) c(m, 1)+c(m, 2) c(m-1,1)}{2 c(m-1,1) c(m, 1) c(1,2)} \\
& +\frac{c(m-2, m) c(m-1,1)-c(m-2,1) c(m-1, m)}{2 c(m-2, m-1) c(m-1, m) c(m-1,1)}+\frac{c(m-1,1)}{2 c(m-1, m) c(m, 1)} \\
& =-\frac{1}{c(m-1, m)}+\frac{1}{c(m-1,1)}-\frac{1}{c(m, 1)}+\frac{c(1,2) c(m-1, m)}{2 c(m-1,1) c(m, 1) c(1,2)} \\
& +\frac{c(1, m) c(m-2, m-1)}{2 c(m-2, m-1) c(m-1, m) c(m-1,1)}+\frac{c(m-1,1)}{2 c(m-1, m) c(m, 1)} \\
& =-\frac{1}{c(m-1, m)}+\frac{1}{c(m-1,1)}-\frac{1}{c(m, 1)}+\frac{c(m-1, m)}{2 c(m-1,1) c(m, 1)} \\
& +\frac{c(1, m)}{2 c(m-1, m) c(m-1,1)}+\frac{c(m-1,1)}{2 c(m-1, m) c(m, 1)} \\
& =\frac{1}{2 c(m-1, m) c(m-1,1) c(m, 1)}\{-2 c(m-1,1) c(m, 1)+2 c(m-1, m) c(m, 1) \\
& \left.-2 c(m-1, m) c(m-1,1)+c(m-1, m)^{2}+c(m, 1)^{2}+c(m-1,1)^{2}\right\} \\
& =\frac{(c(m-1, m)+c(m, 1)-c(m-1,1))^{2}}{2 c(m-1, m) c(m-1,1) c(m, 1)}=0 \text {. }
\end{aligned}
$$

Thus, $J=K$. Proposition 5 is proved.

(Q.E.D.) 
Let $C$ be a semi- $C$-system with $\mu(C)=m$. We shall show that (7) holds for $C$ and (6) holds when $C$ is a $C$-system. For this purpose we define a oneparameter family of semi-C-systems $C(t)$ with $\mu(C(t))=m$.

Definition. Let $t$ be a real number. We define

$$
\tilde{C}(t)=\langle\tilde{c}(i, j ; t): 1 \leq i, j \leq m\rangle
$$

as follows.

(i) $\tilde{c}(i, j ; t)=c(i, j)$, for $1 \leq i, j \leq m-2$.

(ii) $\tilde{c}(m-1, m-1 ; t)=\tilde{c}(m, m ; t)=0$.

(iii) $\tilde{c}(m-1, m ; t)=\tilde{c}(m, m-1 ; t)=c(m-1, m)$.

(iv) For $1 \leq i \leq m-2$,

$$
\tilde{c}(i, m-1 ; t)=\tilde{c}(m-1, i ; t)=\frac{c(i, m-2) t+c(1, i) c(m-2, m-1)}{c(1, m-2)} .
$$

(v) For $1 \leq i \leq m-2$,

$$
\tilde{c}(i, m ; t)=\tilde{c}(m, i ; t)=\frac{c(1, i) c(1, m-1) c(m-2, m)}{c(1, m-2) t}+\frac{c(1, m) c(i, m-2)}{c(1, m-2)}
$$

Let

$$
\begin{aligned}
& r_{1}=\frac{c(1, m-1) c(m-2, m)}{c(m-2, m-1)+c(m-1, m)}, \\
& r_{2}=\frac{c(1,2) c(1, m-1) c(m-2, m)}{c(1, m-2)\{c(m, 1)+c(1,2)-c(m, 2)\}+c(1,2) c(m-2, m)}, \\
& s_{1}=c(m-1, m)+c(m, 1), \\
& s_{2}=\frac{c(1, m-2)\{c(m-3, m-2)+c(m-2, m-1)-c(m-3, m-1)\}+c(1, m-1) c(m-3, m-2)}{c(m-3, m-2)} \\
& a=\max \left(r_{1}, r_{2}\right), \quad b=\min \left(s_{1}, s_{2}\right) .
\end{aligned}
$$

Proposition 6.

(i) $0<a \leq b$.

(ii) For $t \in[a, b], \tilde{C}(t)$ is a semi-C-system.

(iii) If $t=c(1, m-1)$, then $t \in[a, b]$ and $\tilde{C}(t)=C$. 
If $C$ is a C-system, then

(iv) $0<a<b$.

(v) For $t \in(a, b), \tilde{C}(t)$ is a $C$-system.

(vi) If $t=c(1, m-1)$, then $t \in(a, b)$ and $\tilde{C}(t)=C$.

Proof. $0<a$ is obvious. We show that $\tilde{C}(t)$ satisfies the conditions $\mathrm{C} 1, \mathrm{C} 2$ C3. $\mathrm{C} 1$ and $\mathrm{C} 2$ are obvious from the definition.

ProOF OF C3: Let $1 \leq i<j<k<\ell \leq m$. We show that

$$
\tilde{c}(i, k ; t) c(j, \ell ; t)=\tilde{c}(i, j ; t) \tilde{c}(k, \ell ; t)+\tilde{c}(i, \ell ; t) \tilde{c}(j, k ; t) .
$$

If $\ell \leq m-2$, this is obvious, since then

$$
\begin{gathered}
\tilde{c}(i, k ; t)=c(i, k), \quad \tilde{c}(j, \ell ; t)=c(j, \ell), \quad \tilde{c}(i, j ; t)=c(i, j) \\
\tilde{c}(k, \ell ; t)=c(k, \ell), \quad \tilde{c}(i, \ell ; t)=c(i, \ell), \quad \tilde{c}(j, k ; t)=c(j, k) .
\end{gathered}
$$

Next suppose that $\ell=m-1$. Then, $1 \leq i<j<k \leq m-2$. So,

$$
\begin{aligned}
& \tilde{c}(i, k ; t)=c(i, k), \quad \tilde{c}(i, j ; t)=c(i, j), \quad \tilde{c}(j, k ; t)=c(j, k), \\
& \tilde{c}(j, \ell ; t)=\frac{c(j, m-2) t+c(1, j) c(m-2, m-1)}{c(1, m-2)} \\
& \tilde{c}(k, \ell ; t)=\frac{c(k, m-2) t+c(1, k) c(m-2, m-1)}{c(1, m-2)} \\
& \tilde{c}(i, \ell ; t)=\frac{c(i, m-2) t+c(1, i) c(m-2, m-1)}{c(1, m-2)} .
\end{aligned}
$$

Hence,

$$
\begin{gathered}
\tilde{c}(i, j ; t) \tilde{c}(k, \ell ; t)+\tilde{c}(i, \ell ; t) \tilde{c}(j, k ; t)-\tilde{c}(i, k ; t) \tilde{c}(j, \ell ; t) \\
=c(i, j) \frac{c(k, m-2) t+c(1, k) c(m-2, m-1)}{c(1, m-2)} \\
+c(j, k) \frac{c(i, m-2) t+c(1, i) c(m-2, m-1)}{c(1, m-2)} \\
+c(i, k) \frac{c(j, m-2) t+c(1, j) c(m-2, m-1)}{c(1, m-2)}
\end{gathered}
$$




$$
\begin{aligned}
= & \frac{\{c(i, j) c(k, m-2)+c(j, k) c(i, m-2)-c(i, k) c(j, m-2)\} t}{c(1, m-2)} \\
& +\frac{\{c(i, j) c(1, k)+c(j, k) c(1, i)-c(i, k) c(1, j)\}}{c(1, m-2)}=0,
\end{aligned}
$$

since

$$
\begin{gathered}
c(i, j) c(k, m-2)+c(j, k) c(i, m-2)-c(i, k) c(j, m-2)=0 \\
c(i, j) c(1, k)+c(j, k) c(1, i)-c(i, k) c(1, j)=0
\end{gathered}
$$

Note that the last equalities hold also when $k=m-2$ or $i=1$.

Next suppose that $\ell=m$ and $k \leq m-2$. Then

$$
\begin{aligned}
& \tilde{c}(i, k ; t)=c(i, k), \quad \tilde{c}(i, j, t)=c(i, j), \quad \tilde{c}(j, k ; t)=c(j, k) \\
& \tilde{c}(j, \ell ; t)=\frac{c(1, j) c(1, m-1) c(m-2, m)}{c(1, m-2) t}+\frac{c(1, m) c(j, m-2)}{c(1, m-2)} \\
& \tilde{c}(k, \ell ; t)=\frac{c(1, k) c(1, m-1) c(m-2, m)}{c(1, m-2) t}+\frac{c(1, m) c(k, m-2)}{c(1, m-2)} \\
& \tilde{c}(i, \ell ; t)=\frac{c(1, i) c(1, m-1) c(m-2, m)}{c(1, m-2) t}+\frac{c(1, m) c(i, m-2)}{c(1, m-2)}
\end{aligned}
$$

Hence

$$
\begin{aligned}
& \tilde{c}(i, j ; t) \tilde{c}(k, \ell ; t)+\tilde{c}(i, \ell ; t) \tilde{c}(j, k ; t)-\tilde{c}(i, k ; t) \tilde{c}(j, \ell ; t) \\
&= c(i, j)\left\{\frac{c(1, k) c(1, m-1) c(m-2, m)}{c(1, m-2) t}+\frac{c(1, m) c(k, m-2)}{c(1, m-2)}\right\} \\
&+c(j, k)\left\{\frac{c(1, i) c(1, m-1) c(m-2, m)}{c(1, m-2) t}+\frac{c(1, m) c(i, m-2)}{c(1, m-2)}\right\} \\
&-c(i, k)\left\{\frac{c(1, j) c(1, m-1) c(m-2, m)}{c(1, m-2) t}+\frac{c(1, m) c(j, m-2)}{c(1, m-2)}\right\} \\
&= \frac{c(1, m-1) c(m-2, m)}{c(1, m-2) t}\{c(i, j) c(1, k)+c(j, k) c(1, i)-c(1, k) c(1, j)\} \\
&+\frac{c(1, m)}{c(1, m-2)}\{c(i, j) c(k, m-2)+c(j, k) c(i, m-2)-c(i, k) c(j, m-2)\} \\
&= 0 .
\end{aligned}
$$


Next suppose that $\ell=m$ and $k=m-1$. Then

$$
\begin{aligned}
\tilde{c}(i, j ; t) & =c(i, j), \quad \tilde{c}(k, \ell ; t)=c(m-1, m) \\
\tilde{c}(i, k, t) & =\frac{c(i, m-2) t+c(1, i) c(m-2, m-1)}{c(1, m-2)} \\
\tilde{c}(i, \ell ; t) & =\frac{c(1, i) c(1, m-1) c(m-2, m)}{c(1, m-2) t}+\frac{c(1, m) c(i, m-2)}{c(1, m-2)} \\
\tilde{c}(j, k, t) & =\frac{c(j, m-2) t+c(1, j) c(m-2, m-1)}{c(1, m-2)} \\
\tilde{c}(j, \ell ; t) & =\frac{c(1, j) c(1, m-1) c(m-2, m)}{c(1, m-2) t}+\frac{c(1, m) c(j, m-2)}{c(1, m-2)}
\end{aligned}
$$

Hence

$$
\begin{aligned}
\tilde{c}(i, j ; & t) \tilde{c}(k, \ell ; t)+\tilde{c}(i, \ell ; t) \tilde{c}(j, k ; t)-\tilde{c}(i, k ; t) \tilde{c}(j, \ell ; t) \\
= & c(i, j) c(m-1, m)+\left\{\frac{c(1, i) c(1, m-1) c(m-2, m)}{c(1, m-2) t}+\frac{c(1, m) c(i, m-2)}{c(1, m-2)}\right\} \\
& \times\left\{\frac{c(j, m-2) t+c(1, j) c(m-2, m-1)}{c(1, m-2)}\right\} \\
& -\left\{\frac{c(i, m-2) t+c(1, i) c(m-2, m-1)}{c(1, m-2)}\right\} \\
& \times\left\{\frac{c(1, j) c(1, m-1) c(m-2, m)}{c(1, m-2) t}+\frac{c(1, m) c(j, m-2)}{c(1, m-2)}\right\} \\
= & \frac{1}{c(1, m-2)^{2} t}\left[c(i, j) c(m-1, m) c(1, m-2)^{2} t\right. \\
& +\{c(1, m) c(i, m-2) t+c(1, i) c(1, m-1) c(m-2, m)\} \\
& \times\{c(j, m-2) t+c(1, j) c(m-2, m-1)\} \\
& -\{c(1, m) c(j, m-2) t+c(1, j) c(1, m-1) c(m-2, m)\} \\
& \times\{c(i, m-2) t+c(1, i) c(m-2, m-1)\}] \\
= & \frac{1}{c(1, m-2)^{2} t}\left(P t^{2}+Q t+R\right),
\end{aligned}
$$


where

$$
\begin{aligned}
P= & c(1, m) c(i, m-2) c(j, m-2)-c(1, m) c(j, m-2) c(i, m-2)=0 \\
Q= & c(i, j) c(m-1, m) c(1, m-2)^{2}+c(1, m) c(i, m-2) c(1, j) c(m-2, m-1) \\
& +c(1, i) c(1, m-1) c(m-2, m) c(j, m-2) \\
& -c(1, m) c(j, m-2) c(1, i) c(m-2, m-1) \\
& -c(1, j) c(1, m-1) c(m-2, m) c(i, m-2) \\
= & c(i, j) c(m-1, m) c(1, m-2)^{2}+c(1, m) c(m-2, m-1)\{c(i, m-2) c(1, j) \\
& -c(j, m-2) c(1, i)\}-c(1, m-1) c(m-2, m)\{c(1, j) c(i, m-2) \\
& -c(1, i) c(j, m-2)\} \\
= & c(i, j) c(m-1, m) c(1, m-2)^{2}+c(1, m) c(m-2, m-1) c(1, m-2) c(i, j) \\
& -c(1, m-1) c(m-2, m) c(1, m-2) c(i, j) \\
= & \quad(i, j) c(1, m-2)\{c(m-1, m) c(1, m-2)+c(1, m) c(m-2, m-1) \\
& -c(1, m-1) c(m-2, m)\} \\
= & 0, \\
& \quad c(1, i) c(1, m-1) c(m-2, m) c(1, j) c(m-2, m-1) c(1, m-1) c(m-2, m) c(1, i) c(m-2, m-1)=0 . \\
& \\
& \\
& \\
& \\
& \\
&
\end{aligned}
$$

Thus, C3 is proved.

Next suppose that $t=c(1, m-1)$. Then, for $1 \leq i \leq m-2$,

$$
\begin{aligned}
\tilde{c}(i, m-1 ; t) & =\tilde{c}(m-1, i ; t)=\frac{c(i, m-2) c(1, m-1)+c(1, i) c(m-2, m-1)}{c(1, m-2)} \\
& =\frac{c(1, m-2) c(i, m-1)}{c(1, m-2)}=c(i, m-1)
\end{aligned}
$$




$$
\begin{aligned}
\tilde{c}(i, m ; t) & =\tilde{c}(m, i ; t) \\
& =\frac{c(1, i) c(1, m-1) c(m-2, m)}{c(1, m-2) c(1, m-1)}+\frac{c(1, m) c(i, m-2)}{c(1, m-2)} \\
& =\frac{c(1, i) c(m-2, m)+c(1, m) c(i, m-2)}{c(1, m-2)} \\
& =\frac{c(1, m-2) c(i, m)}{c(1, m-2)}=c(i, m) .
\end{aligned}
$$

Thus, $\tilde{C}(t)=C$, when $t=c(1, m-1)$.

Next by $\mathrm{C}^{\prime}$,

$$
c(m-2, m) \leq c(m-2, m-1)+c(m-1, m) .
$$

Hence,

$$
c(1, m-1) c(m-2, m) \leq c(1, m-1)\{c(m-2, m-1)+c(m-1, m)\} .
$$

Hence,

$$
\frac{c(1, m-1) c(m-2, m)}{c(m-2, m-1)+c(m-1, m)} \leq c(1, m-1),
$$

that is, $r_{1} \leq c(1, m-1)$.

Also by $\mathrm{C}^{\prime}$,

$$
c(m, 1)+c(1,2) \geq c(m, 2) .
$$

Hence,

$$
\begin{aligned}
c(1,2) c(1, m-1) c(m-2, m) \leq & c(1, m-2)\{c(m, 1)+c(1,2)-c(m, 2)\} \\
& +c(1,2) c(1, m-1) c(m-2, m) .
\end{aligned}
$$

Hence,

$$
\frac{c(1,2) c(1, m-1) c(m-2, m)}{c(1, m-2)\{c(m, 1)+c(1,2)-c(m, 2)\}+c(1,2) c(m-2, m)} \leq c(1, m-1),
$$

that is, $r_{2} \leq c(1, m-1)$.

Also by $\mathrm{C} 4^{\prime}$,

$$
c(1, m-1) \leq c(m-1, m)+c(m, 1) .
$$

Hence, $c(1, m-1) \leq s_{1}$. 
Also by $\mathrm{C}^{\prime}$,

$$
c(m-3, m-2)+c(m-2, m-1) \geq c(m-3, m-1) .
$$

Hence,

$$
\begin{aligned}
& c(1, m-1) c(m-3, m-2) \\
& \leq c(1, m-2)\{c(m-3, m-2)+c(m-2, m-1)-c(m-3, m-1)\} \\
& \quad+c(1, m-1) c(m-3, m-2) .
\end{aligned}
$$

Hence,

$$
\begin{aligned}
& c(1, m-1) \\
& \quad \leq \frac{c(1, m-2)\{c(m-3, m-2)+c(m-2, m-1)-c(m-3, m-1)\}+c(1, m-1) c(m-3, m-2)}{c(m-3, m-2)},
\end{aligned}
$$

that is, $c(1, m-1) \leq s_{2}$.

Therefore,

$$
a=\max \left(r_{1}, r_{2}\right) \leq c(1, m-1) \leq \min \left(s_{1}, s_{2}\right)=b .
$$

Hence, $a \leq b$ and $c(1, m-1) \in[a, b]$.

Thus, (i) and (iii) of Proposition 6 are proved.

Next we shall show that $\mathrm{C}^{\prime}$ holds for $C(t)$ with $t \in[a, b]$, that is,

$$
\begin{gathered}
\tilde{c}(m, 1 ; t)+\tilde{c}(1,2 ; t) \geq \tilde{c}(m, 2 ; t) \\
\tilde{c}(i-1, i ; t)+\tilde{c}(i, i+1 ; t) \geq \tilde{c}(i-1, i+1 ; t)
\end{gathered}
$$

for $2 \leq i \leq m-1$, and

$$
\tilde{c}(m-1, m ; t)+\tilde{c}(m, 1 ; t) \geq \tilde{c}(m-1,1 ; t)
$$

First we prove (8). Now,

$$
\begin{aligned}
& \tilde{c}(m, 1 ; t)=c(m, 1), \quad \tilde{c}(1,2 ; t)=c(1,2) \\
& \tilde{c}(m, 2 ; t)=\frac{c(1,2) c(1, m-1) c(m-2, m)}{c(1, m-2) t}+\frac{c(1, m) c(2, m-2)}{c(1, m-2)}
\end{aligned}
$$


Hence,

$$
\begin{aligned}
\tilde{c}( & m, 1 ; t)+\tilde{c}(1,2 ; t)-\tilde{c}(m, 2 ; t) \\
= & c(m, 1)+c(1,2)-\frac{c(1,2) c(1, m-1) c(m-2, m)}{c(1, m-2) t}-\frac{c(1, m) c(2, m-2)}{c(1, m-2)} \\
= & \frac{\{(c(m, 1)+c(1,2)) c(1, m-2)-c(1, m) c(2, m-2)\} t-c(1,2) c(1, m-1) c(m-2, m)}{c(1, m-2) t} \\
= & \frac{1}{c(1, m-2) t}[\{(c(m, 1)+c(1,2)) c(1, m-2)-c(1, m-2) c(2, m) \\
& +c(1,2) c(m-2, m)\} t-c(1,2) c(1, m-1) c(m-2, m)] \\
= & \frac{1}{c(1, m-2) t}[\{(c(m, 1)+c(1,2)-c)(2, m)) c(1, m-2)+c(1,2) c(m-2, m)\} t \\
& -c(1,2) c(1, m-1) c(m-2, m)] \\
= & \frac{\{c(m, 1)+c(1,2)-c(2, m)) c(1, m-2)+c(1,2) c(m-2, m)\}}{c(1, m-2) t} \\
& \times\left\{t-\frac{c(1,2) c(1, m-1) c(m-2, m)}{(c(m, 1)+c(1,2)-c(2, m)) c(1, m-2)+c(1,2) c(m-2, m)}\right\} \\
= & \frac{\{(c(m, 1)+c(1,2)-c(2, m)) c(1, m-2)+c(1,2) c(m-2, m)\}}{c(1, m-2) t}\left(t-r_{2}\right) \geq 0 . \\
& \\
& \\
&
\end{aligned}
$$

Next we prove (9). (9) for $i \leq m-3$ is obvious, since then

$$
\begin{gathered}
\tilde{c}(i-1, i ; t)=c(i-1, i), \quad \tilde{c}(i, i+1 ; t)=c(i, i+1), \\
\tilde{c}(i-1, i+1 ; t)=c(i-1, i+1) .
\end{gathered}
$$

When $i=m-2$, (9) becomes

$$
\tilde{c}(m-3, m-2 ; t)+\tilde{c}(m-2, m-1 ; t) \geq c(m-3, m-1 ; t) .
$$

Now,

$$
\begin{gathered}
\tilde{c}(m-3, m-2 ; t)=c(m-3, m-2), \quad \tilde{c}(m-2, m-1 ; t)=c(m-2, m-1), \\
\tilde{c}(m-3, m-1 ; t)=\frac{c(m-3, m-2) t+c(1, m-3) c(m-2, m-1)}{c(1, m-2)} .
\end{gathered}
$$


Hence,

$$
\begin{aligned}
\tilde{c}( & -3, m-2 ; t)+\tilde{c}(m-2, m-1 ; t)-\tilde{c}(m-3, m-1 ; t) \\
= & c(m-3, m-2)+c(m-2, m-1)-\frac{c(m-3, m-2) t+c(1, m-3) c(m-2, m-1)}{c(1, m-2)} \\
= & \frac{\{c(m-3, m-2)+c(m-2, m-1)\} c(1, m-2)-c(1, m-3) c(m-2, m-1)-c(m-3, m-2) t}{c(1, m-2)} \\
= & \frac{1}{c(1, m-2)}[\{c(m-3, m-2)+c(m-2, m-1)\} c(1, m-2)-c(1, m-2) c(m-1, m-3) \\
& +c(1, m-1) c(m-3, m-2)-c(m-3, m-2) t] \\
= & \frac{1}{c(1, m-2)}[c(1, m-2)\{c(m-3, m-2)+c(m-2, m-1)-c(m-3, m-1)\} \\
& +c(1, m-1) c(m-3, m-2)-c(m-3, m-2) t] \\
= & \frac{c(m-3, m-2)}{c(1, m-2)} \\
& \quad \times\left[\frac{c(1, m-2)\{c(m-3, m-2)+c(m-2, m-1)-c(m-3, m-1)\}+c(1, m-1) c(m-3, m-2)}{c(m-3, m-2)}-t\right] \\
= & \frac{c(m-3, m-2)}{c(1, m-2)}\left(s_{2}-t\right) \geq 0 .
\end{aligned}
$$

When $i=m-1$, (9) becomes

$$
\tilde{c}(m-2, m-1 ; t)+\tilde{c}(m-1, m ; t) \geq \tilde{c}(m-2, m ; t) .
$$

Now,

$$
\begin{gathered}
\tilde{c}(m-2, m-1 ; t)=c(m-2, m-1), \quad \tilde{c}(m-1, m ; t)=c(m-1, m), \\
\tilde{c}(m-2, m ; t)=\frac{c(1, m-2) c(1, m-1) c(m-2, m)}{c(1, m-2) t}+\frac{c(1, m) c(m-2, m-2)}{c(1, m-2)} \\
=\frac{c(1, m-1) c(m-2, m)}{t} .
\end{gathered}
$$

Hence,

$$
\begin{aligned}
\tilde{c}(m & -2, m-1 ; t)+\tilde{c}(m-1, m ; t)-\tilde{c}(m-2, m ; t) \\
& =c(m-2, m-1)+c(m-1, m)-\frac{c(1, m-1) c(m-2, m)}{t} \\
& =\frac{\{c(m-2, m-1)+c(m-1, m)\}}{t}\left\{t-\frac{c(1, m-1) c(m-2, m)}{c(m-2, m-1)+c(m-1, m)}\right\} \\
& =\frac{\{c(m-2, m-1)+c(m-1, m)\}}{t}\left(t-r_{1}\right) \geq 0 .
\end{aligned}
$$


Next we prove (10). Now,

$$
\tilde{c}(m-1, m ; t)=c(m-1, m), \quad \tilde{c}(m, 1 ; t)=c(m, 1), \quad c(m-1,1 ; t)=t .
$$

Hence,

$$
\begin{aligned}
\tilde{c}(m-1, m ; t)+\tilde{c}(m, 1 ; t)-\tilde{c}(m-1,1 ; t) & =c(m-1, m)+c(m, 1)-t \\
& =s_{1}-t \geq 0
\end{aligned}
$$

Next suppose that $C$ is a $C$-system. Then by the above proof we have $r_{1}<c(1, m-1), \quad r_{2}<c(1, m-1), \quad c(1, m-1)<s_{1}, \quad c(1, m-1)<s_{2}$.

Hence,

$$
a=\max \left(r_{1}, r_{2}\right)<c(1, m-1)<\min \left(s_{1}, s_{2}\right)=b .
$$

Thus, (iv) and (vi) of Proposition 6 is proved.

Moreover, similarly to the proof of (8), (9), (10) above we can show that, for $t \in(a, b)$,

$$
\begin{aligned}
\tilde{c}(m, 1 ; t)+\tilde{c}(1,2 ; t) & >\tilde{c}(m, 2 ; t) \\
\tilde{c}(i-1, i ; t)+\tilde{c}(i, i+1 ; t) & >\tilde{c}(i-1, i+1 ; t) \quad(2 \leq i \leq m-1),
\end{aligned}
$$

and

$$
\tilde{c}(m-1, m ; t)+\tilde{c}(m, 1 ; t)>\tilde{c}(m-1,1 ; t) .
$$

Thus, (v) of Proposition 6 is proved.

(Q.E.D.)

Let

$$
\begin{aligned}
f(t)= & \left\{\sum_{i=1}^{m-1} \tilde{c}(i, i+1 ; t)+\tilde{c}(m, 1 ; t)\right\}\left\{\sum_{i=1}^{m-1} \frac{1}{\tilde{c}(i, i+1 ; t)}+\frac{1}{\tilde{c}(m, 1 ; t)}\right. \\
& -\frac{\tilde{c}(m, 2 ; t)}{2 \tilde{c}(m, 1 ; t) \tilde{c}(1,2 ; t)}-\sum_{i=2}^{m-1} \frac{\tilde{c}(i-1, i+1 ; t)}{2 \tilde{c}(i-1, i ; t) \tilde{c}(i, i+1 ; t)} \\
& \left.-\frac{\tilde{c}(m-1,1 ; t)}{2 \tilde{c}(m-1, m ; t) \tilde{c}(m, 1 ; t)}\right\}-4 .
\end{aligned}
$$

Then,

Proposition 7. Let $C$ be a semi-C-system. If $t \in[a, b]$, then, $f^{\prime \prime}(t)<0$. 
ProOf. $\quad \tilde{c}(i, i+1 ; t)=c(i, i+1) \quad(i=1, \ldots, m-1) \quad$ and $\quad \tilde{c}(m, 1 ; t)=c(m, 1)$ are constants. Also, $\tilde{c}(i-1, i+1 ; t)=c(i-1, i+1)(2 \leq i \leq m-3)$ are constants. Moreover,

$$
\begin{aligned}
\tilde{c}(m-3, m-1 ; t) & =\frac{c(m-3, m-2) t+c(1, m-3) c(m-2, m-1)}{c(1, m-2)} \\
\tilde{c}(m-2, m ; t) & =\frac{c(1, m-1) c(m-2, m)}{t} \\
\tilde{c}(m-1,1 ; t) & =t \\
\tilde{c}(m, 2 ; t) & =\frac{c(1,2) c(1, m-1) c(m-2, m)}{c(1, m-2) t}+\frac{c(1, m) c(2, m-2)}{c(1, m-2)} .
\end{aligned}
$$

Hence,

$$
\begin{aligned}
f^{\prime \prime}(t)= & \left\{\sum_{i=1}^{m-1} c(i, i+1)+c(m, 1)\right\}\left\{-\frac{\tilde{c}(m, 2 ; t)^{\prime \prime}}{2 c(m, 1) c(1,2)}\right. \\
& -\frac{\tilde{c}(m-3, m-1 ; t)^{\prime \prime}}{2 c(m-3, m-2) c(m-2, m-1)}-\frac{\hat{c}(m-2, m ; t)^{\prime \prime}}{2 c(m-2, m-1) c(m-1, m)} \\
& \left.-\frac{\tilde{c}(m-1,1 ; t)^{\prime \prime}}{2 c(m-1, m) c(m, 1)}\right\} \\
= & \left\{\sum_{i=1}^{m-1} c(i, i+1)+c(m, 1)\right\}\left\{-\frac{c(1,2) c(1, m-1) c(m-2, m)}{2 c(m, 1) c(1,2) c(1, m-1)} \frac{2}{t^{3}}\right. \\
& \left.-\frac{c(1, m-1) c(m-2, m)}{2 c(m-2, m-1) c(m-1, m)} \frac{2}{t^{3}}\right\}<0 .
\end{aligned}
$$

Proposition 8. If $f^{\prime \prime}(t)<0$ for each $t \in[a, b]$, then $f(t)>\min \{f(a), f(b)\}$, for each $t \in(a, b)$.

Proof. Suppose that $f^{\prime \prime}(t)<0$ for each $t \in[a, b]$. Then, $f^{\prime}(t)$ is strictly decreasing in $[a, b]$.

Case 1. $f^{\prime}(b)<f^{\prime}(a) \leq 0$.

Then, $f^{\prime}(t)<0$ for each $t \in(a, b)$. Hence, $f(t)$ is strictly decreasing in $[a, b]$. Hence,

$$
f(t)>f(b) \geq \min \{f(a), f(b)\}
$$

for each $t \in(a, b)$. 
Case 2. $0 \leq f^{\prime}(b)<f^{\prime}(a)$.

Then, $f^{\prime}(t)>0$ for each $t \in(a, b)$. Hence, $f(t)$ is strictly increasing in $[a, b]$. Hence,

$$
f(t)>f(a) \geq \min \{f(a), f(b)\}
$$

for each $t \in(a, b)$.

Case 3. $f^{\prime}(b)<0<f^{\prime}(a)$.

Then, there is a unique $d \in(a, b)$ such that $f^{\prime}(d)=0 . f(t)$ is strictly increasing in $[a, d]$ and strictly decreasing in $[d, b]$. So, if $t \in(a, d]$, then

$$
f(t)>f(a) \geq \min \{f(a), f(b)\},
$$

and if $t \in[d, b)$, then

$$
f(t)>f(b) \geq \min \{f(a), f(b)\} .
$$

This completes the proof of Proposition 8.

(Q.E.D.)

Now we can show that (7) holds for the semi- $C$-system $C$. Since $\tilde{C}(c(1, m-1))=C$, it suffices to show that $f(t) \geq 0$, for $t \in[a, b]$. By Proposition 7 and 8 , it suffices to show that $f(a) \geq 0$ and $f(b) \geq 0$.

$a=\max \left\{r_{1}, r_{2}\right\}$. So, $a=r_{1}$ or $a=r_{2}$.

Case 1. $a=r_{1}$.

Then, $\tilde{C}\left(r_{1}\right)$ is a semi- $C$-system such that $\mu\left(\tilde{C}\left(r_{1}\right)\right)=m$ and

$$
\begin{aligned}
\tilde{c}\left(m-2, m ; r_{1}\right)= & \frac{c(1, m-2) c(1, m-1) c(m-2, m)}{c(1, m-2) r_{1}}+\frac{c(1, m) c(m-2, m-2)}{c(1, m-2)} \\
& =\frac{c(1, m-1) c(m-2, m)}{r_{1}}=c(m-2, m-1)+c(m-1, m) \\
& =\tilde{c}\left(m-2, m-1 ; r_{1}\right)+\tilde{c}\left(m-1, m ; r_{1}\right) .
\end{aligned}
$$

Hence, by Proposition 5, (7) holds for $\tilde{C}\left(r_{1}\right)$, that is, $f\left(r_{1}\right) \geq 0$.

Cases 2. $a=r_{2}$.

Then, $\tilde{C}\left(r_{2}\right)$ is a semi- $C$-system such that $\mu\left(\tilde{C}\left(r_{2}\right)\right)=m$. Now,

$$
\begin{aligned}
\tilde{c}\left(m, 2 ; r_{2}\right)= & \frac{c(1,2) c(1, m-1) c(m-2, m)}{c(1, m-2) r_{2}}+\frac{c(1, m) c(2, m-2)}{c(1, m-2)} \\
= & \frac{c(1, m-2)\{c(m, 1)+c(1,2)-c(m, 2)\}+c(1,2) c(m-1, m)}{c(1, m-2)} \\
& +\frac{c(1, m) c(2, m-2)}{c(1, m-2)}
\end{aligned}
$$




$$
\begin{aligned}
& =c(m, 1)+c(1,2)-c(m, 2)+\frac{c(1,2) c(m-2, m)+c(1, m) c(2, m-2)}{c(1, m-2)} \\
& =c(m, 1)+c(1,2)-c(m, 2)+\frac{c(1, m-2) c(2, m)}{c(1, m-2)} \\
& =c(m, 1)+c(1,2) \\
& =\tilde{c}\left(m, 1 ; r_{2}\right)+\tilde{c}\left(1,2 ; r_{2}\right) .
\end{aligned}
$$

Hence, by Proposition 5, (7) holds for $\tilde{C}\left(r_{2}\right)$, that is $f\left(r_{2}\right) \geq 0$.

Thus, we have proved that $f(a) \geq 0$. Next we prove that $f(b) \geq 0 . b=$ $\min \left\{s_{1}, s_{2}\right\}$. So, $b=s_{1}$ or $b=s_{2}$.

Case 1. $b=s_{1}$.

Then, $\tilde{C}\left(s_{1}\right)$ is a semi- $C$-system such that $\mu\left(\tilde{C}\left(s_{1}\right)\right)=m$ and

$$
\begin{aligned}
\tilde{c}\left(1, m-1 ; s_{1}\right) & =s_{1}=c(m-1, m)+c(m, 1) \\
& =\tilde{c}\left(m-1, m ; s_{1}\right)+\tilde{c}\left(m, 1 ; s_{1}\right) .
\end{aligned}
$$

Hence, by Proposition 5, (7) holds for $\tilde{C}\left(s_{1}\right)$, that is $f\left(s_{1}\right) \geq 0$.

Case 2. $b=s_{2}$.

Then, $\tilde{C}\left(s_{2}\right)$ is a semi-C-system such that $\mu\left(\tilde{C}\left(s_{2}\right)\right)=m$ and

$$
\begin{aligned}
\tilde{c}\left(m-3, m-1 ; s_{2}\right)= & \left\{c(m-3, m-2) s_{2}+c(1, m-3) c(m-2, m-1)\right\} / c(1, m-2) \\
= & {[c(1, m-2)\{c(m-3, m-2)+c(m-2, m-1)-c(m-3, m-1)\}} \\
& +c(1, m-1) c(m-3, m-2) \\
& +c(1, m-3) c(m-2, m-1)] / c(1, m-2) \\
= & c(m-3, m-2)+c(m-2, m-1)-c(m-3, m-1) \\
& +\frac{c(1, m-2) c(m-3, m-1)}{c(1, m-2)} \\
= & c(m-3, m-2)+c(m-2, m-1) \\
= & \tilde{c}\left(m-3, m-2 ; s_{2}\right)+\tilde{c}\left(m-2, m-1 ; s_{2}\right) .
\end{aligned}
$$

Hence by Proposition 5, (7) holds for $\tilde{C}\left(s_{2}\right)$, that is, $f\left(s_{2}\right) \geq 0$. This completes the proof of (7) for the semi- $C$-system $C$. 
Next suppose that $C$ is a $C$-system. Then, by Proposition 6, $\tilde{C}(c(1, m-1))=C$ and $c(1, m-1) \in(a, b)$. Hence by Proposition 7 and 8 ,

$$
f(c(1, m-1))>\min \{f(a), f(b)\} \geq 0 .
$$

Hence $f(c(1, m-1))>0$, that is, (6) holds for $C$. This completes the proof of Lemma 4 and hence of Lemma 3.

ProOF OF THEOREM 1.

Suppose that $\alpha_{i} \beta_{k}-\beta_{i} \alpha_{k} \neq 0(i \neq k), \gamma_{j} \delta_{\ell}-\delta_{j} \gamma_{\ell} \neq 0(j \neq \ell), e>0, f>0$ and that for all $(x, y) \in L$,

$$
\sum_{i=1}^{m}\left|\alpha_{i} x-\beta_{i} y\right| \geq 1 \quad \text { and } \quad \sum_{i=1}^{n}\left|\gamma_{j} x-\delta_{j} y\right| \geq 1
$$

We shall prove that

$$
\sum_{i=1}^{m} \sum_{j=1}^{n}\left|\alpha_{i} \delta_{j}-\beta_{i} \gamma_{j}\right| \geq 1
$$

Now, by Lemma 3, we have

$$
\sum_{i=1}^{m} \sum_{k=1}^{m}\left|\alpha_{i} \beta_{k}-\beta_{i} \alpha_{k}\right| \geq 1 \text { and } \sum_{j=1}^{n} \sum_{\ell=1}^{n}\left|\gamma_{j} \delta_{\ell}-\delta_{j} \gamma_{\ell}\right| \geq 1
$$

So, it suffices to prove that

$$
\left\{\sum_{i=1}^{m} \sum_{j=1}^{n}\left|\alpha_{i} \delta_{j}-\beta_{i} \gamma_{j}\right|\right\}^{2} \geq\left\{\sum_{i=1}^{m} \sum_{k=1}^{m}\left|\alpha_{i} \beta_{k}-\beta_{i} \alpha_{k}\right|\right\}\left\{\sum_{j=1}^{n} \sum_{\ell=1}^{n}\left|\gamma_{j} \delta_{\ell}-\delta_{j} \gamma_{\ell}\right|\right\} .
$$

Moreover, if (12) is proved and if the equality in (11) holds, then by (12) we have

$$
\sum_{i=1}^{m} \sum_{k=1}^{m}\left|\alpha_{i} \beta_{k}-\beta_{i} \alpha_{k}\right|=1 \quad \text { and } \quad \sum_{j=1}^{n} \sum_{\ell=1}^{n}\left|\gamma_{j} \delta_{\ell}-\delta_{j} \gamma_{\ell}\right|=1
$$

By Lemma 3, we must have $m=2, n=2$ and $\left|\alpha_{1} \beta_{2}-\beta_{1} \alpha_{2}\right|=1 / 2,\left|\gamma_{1} \delta_{2}-\delta_{1} \gamma_{2}\right|=$ $1 / 2$. Thus we have only to prove (12).

In order to prove (12), it suffices to prove that the quadratic form 


$$
\begin{aligned}
F\left(x_{1}, \ldots, x_{m}\right)= & \left\{\sum_{i=1}^{m} \sum_{j=1}^{n}\left|\alpha_{i} \delta_{j}-\beta_{i} \gamma_{j}\right| x_{i}\right\}^{2} \\
& -\left\{\sum_{i=1}^{m} \sum_{k=1}^{m}\left|\alpha_{i} \beta_{k}-\beta_{i} \alpha_{k}\right| x_{i} x_{k}\right\}\left\{\sum_{j=1}^{n} \sum_{\ell=1}^{n}\left|\gamma_{j} \delta_{\ell}-\delta_{j} \gamma_{\ell}\right|\right\}
\end{aligned}
$$

is positive semi-definite, i.e. $F\left(x_{1}, \ldots, x_{m}\right) \geq 0$ for every real numbers $x_{1}, \ldots, x_{m}$.

Now, let

$$
\begin{aligned}
\xi_{i k}= & \left\{\sum_{j=1}^{n}\left|\alpha_{i} \delta_{j}-\beta_{i} \gamma_{j}\right|\right\}\left\{\sum_{\ell=1}^{n}\left|\alpha_{k} \delta_{\ell}-\beta_{k} \gamma_{\ell}\right|\right\} \\
& -\left|\alpha_{i} \beta_{k}-\beta_{i} \alpha_{k}\right|\left\{\sum_{j=1}^{n} \sum_{\ell=1}^{n}\left|\gamma_{j} \delta_{\ell}-\delta_{j} \gamma_{\ell}\right|\right\} .
\end{aligned}
$$

Then, $\xi_{i k}=\xi_{k i}$ and

$$
F\left(x_{1}, \ldots, x_{m}\right)=\sum_{i=1}^{m} \sum_{k=1}^{m} \xi_{i k} x_{i} x_{k}
$$

Proposition 9. A quadratic form

$$
G\left(x_{1}, \ldots, x_{m}\right)=\sum_{i=1}^{m} \sum_{k=1}^{m} p_{i k} x_{i} x_{k}
$$

(with $p_{i k}=p_{k i}$ ) is positive semi-definite if and only if

(*) for each sequence $i_{1}, \ldots, i_{r}(r \geq 1)$ of natural numbers such that $1 \leq$ $i_{1}<i_{2}<\cdots<i_{r} \leq m$,

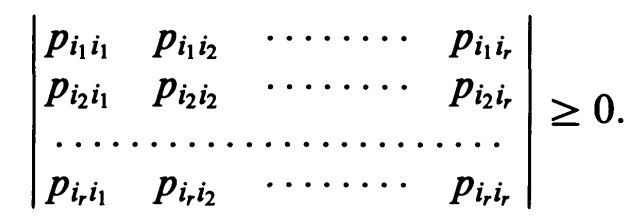

The proof of Proposition 9 on matrix theory is omitted.

By Proposition 9, it suffices to prove that

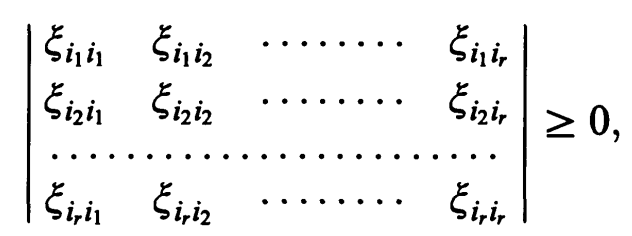


for every sequence $i_{1}, \ldots i_{r}$ such that $1 \leq i_{1}<\cdots<i_{r} \leq m$. By the change of subscript $i_{k}$ to $k$, it suffices to prove that

$$
\left|\begin{array}{ccc}
\xi_{11} & \cdots \cdots & \xi_{1 m} \\
\cdots \cdots & \cdots & \ldots \\
\xi_{m 1} & \cdots \cdots & \xi_{m m}
\end{array}\right| \geq 0
$$

for every $\alpha_{i}, \beta_{i}, \gamma_{j}, \delta_{j}$.

Now, let $a_{i}=\sum_{j=1}^{n}\left|\alpha_{i} \delta_{j}-\beta_{i} \gamma_{j}\right|, \quad d_{i k}=\left|\alpha_{i} \beta_{k}-\beta_{i} \alpha_{k}\right|, \quad t=\sum_{j=1}^{n} \sum_{\ell=1}^{n}\left|\gamma_{j} \delta_{\ell}-\delta_{j} \gamma_{\ell}\right|$. Then, $\xi_{i k}=a_{i} a_{k}-d_{i k}$. Now,

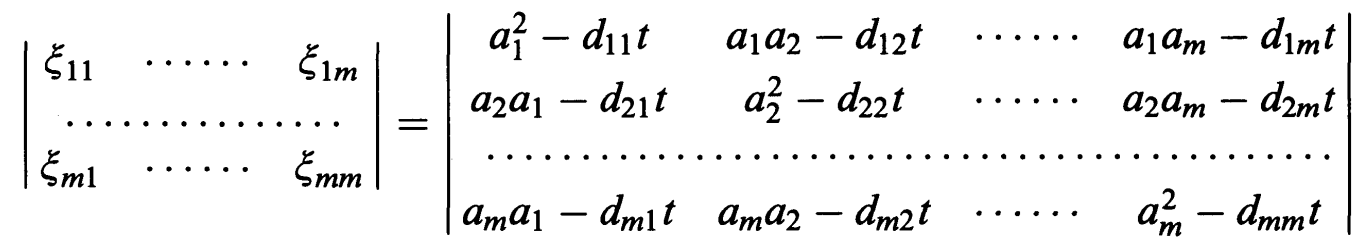

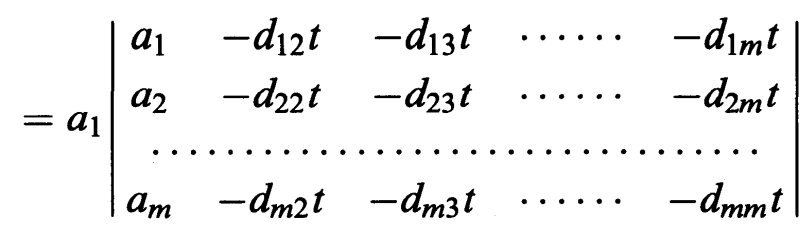

$$
\begin{aligned}
& +a_{2}\left|\begin{array}{ccccc}
-d_{11} t & a_{1} & -d_{13} t & \cdots \cdots & -d_{1 m} t \\
-d_{21} t & a_{2} & -d_{23} t & \cdots \cdots & -d_{2 m} t \\
\cdots \cdots & \ldots & \ldots & \ldots \ldots \ldots & \ldots \\
-d_{m 1} t & a_{m} & -d_{m 3} t & \ldots \ldots & -d_{m m} t
\end{array}\right|
\end{aligned}
$$

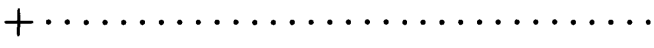

$$
\begin{aligned}
& +a_{m}\left|\begin{array}{ccccc}
-d_{11} t & -d_{12} t & \cdots \cdots & -d_{1 m-1} t & a_{1} \\
-d_{21} t & -d_{22} t & \cdots \cdots & -d_{2 m-1} t & a_{2} \\
\cdots \cdots \cdots & \ldots \ldots \ldots \ldots \ldots \ldots \cdots \cdots \\
-d_{m 1} t & -d_{m 2} t & \cdots \cdots & -d_{m m-1} t & a_{m}
\end{array}\right| \\
& +\left|\begin{array}{ccccc}
-d_{11} t & -d_{12} t & \cdots \cdots & -d_{1 m-1} t & -d_{1 m} t \\
-d_{21} t & -d_{22} t & \cdots \cdots & -d_{2 m-1} t & -d_{2 m} t \\
\cdots \cdots \cdots \cdots \cdots \cdots \cdots \cdots \cdots \cdots \cdots \cdots \cdots & \ldots \cdots \cdots \cdots \\
-d_{m 1} t & -d_{m 2} t & \cdots \cdots & -d_{m m-1} t & -d_{m m} t
\end{array}\right| \\
& =(-1)^{m-1} t^{m-1} a_{1}\left|\begin{array}{ccccc}
a_{1} & d_{12} & d_{13} & \cdots \cdots & d_{1 m} \\
a_{2} & d_{22} & d_{23} & \cdots \cdots & d_{2 m} \\
\cdots & \ldots \ldots & \ldots \cdots \cdots \cdots & \cdots \\
a_{m} & d_{m 2} & d_{m 3} & \cdots \cdots & d_{m m}
\end{array}\right|
\end{aligned}
$$




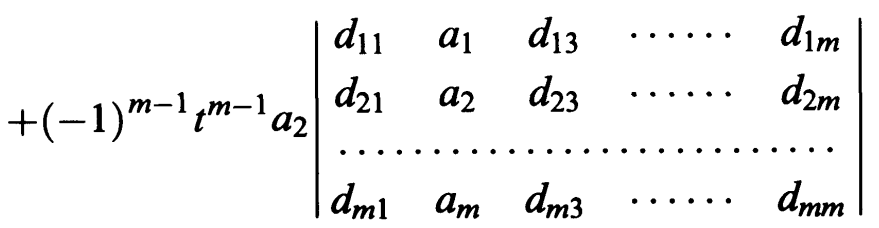

$$
\begin{aligned}
& +\cdots \cdots \cdots \cdots \cdots \cdots \cdots \cdots
\end{aligned}
$$

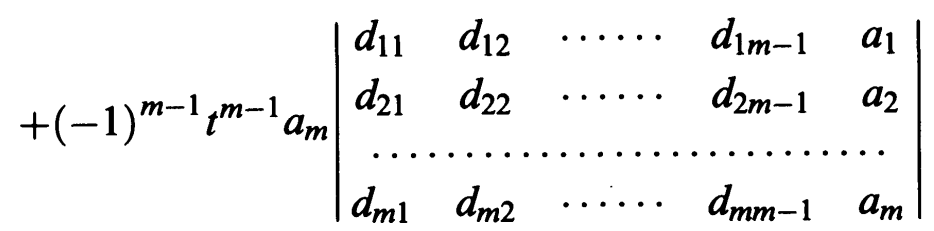

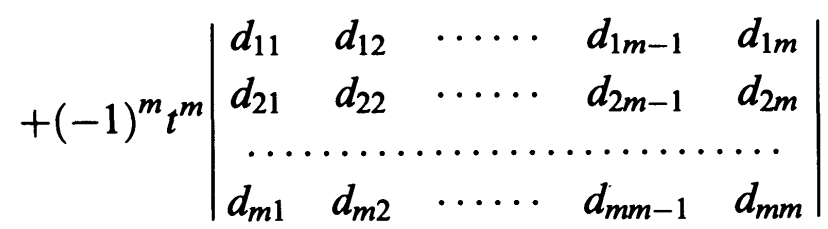

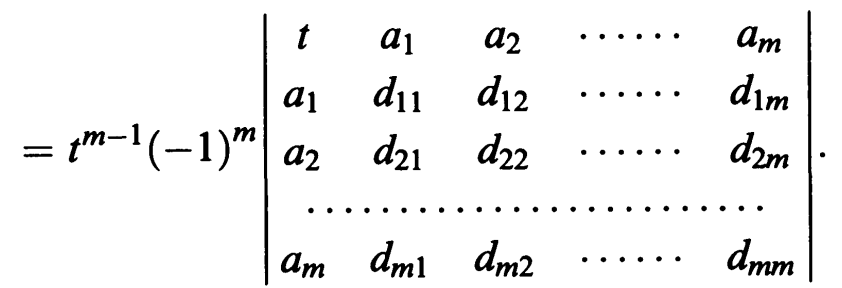

Since $t>0$, it suffices to prove that

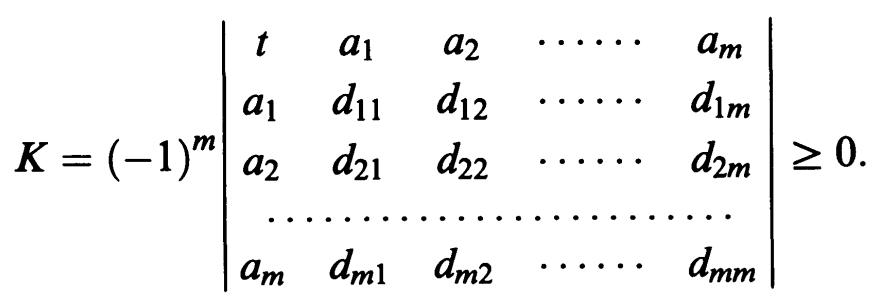

Since

$$
t=\sum_{j=1}^{n}\left(\sum_{\ell=1}^{n}\left|\gamma_{j} \delta_{\ell}-\delta_{j} \gamma_{\ell}\right|\right)
$$

and

$$
a_{i}=\sum_{j=1}^{n}\left|\alpha_{i} \delta_{j}-\beta_{i} \gamma_{j}\right|=\sum_{\ell=1}^{n}\left|\alpha_{i} \delta_{\ell}-\beta_{i} \gamma_{l}\right|
$$


we have

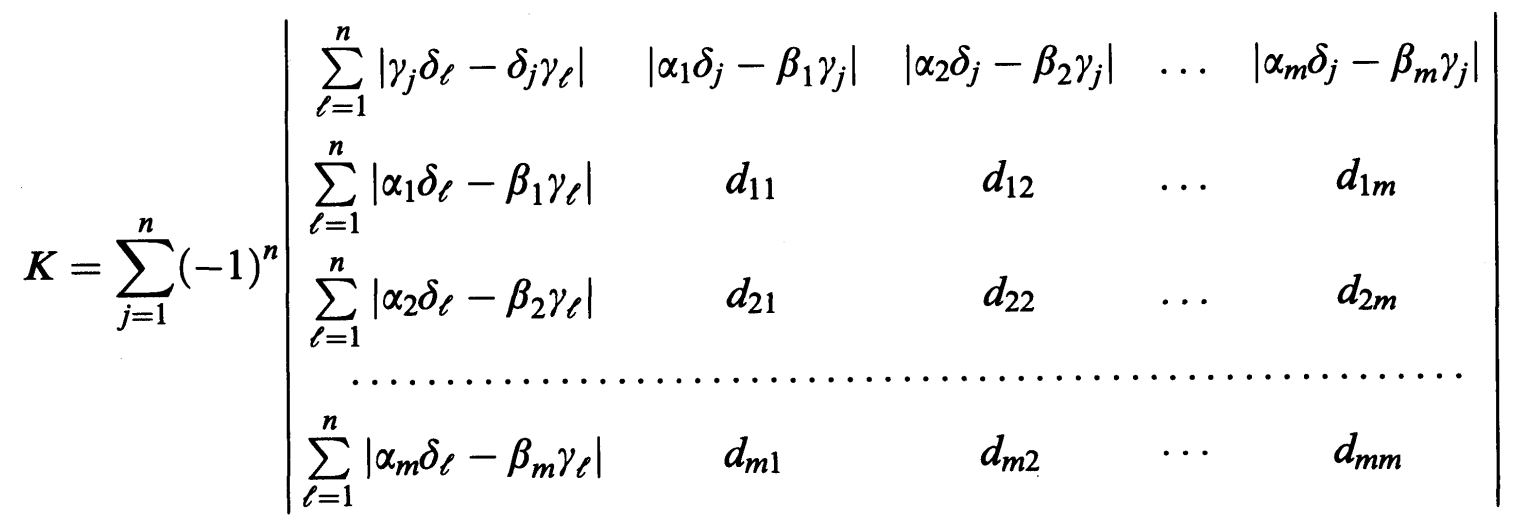

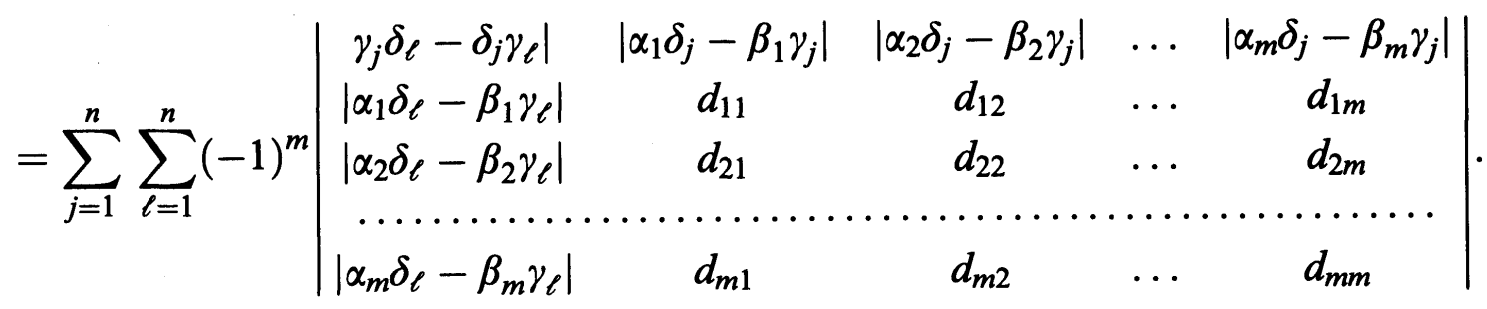

So, it suffices to prove that

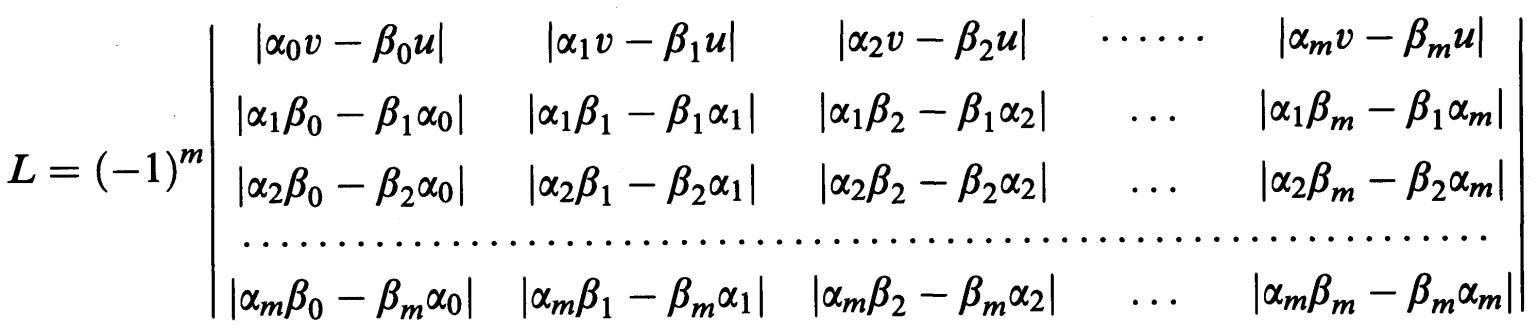

$$
\begin{aligned}
& \geq 0 \text {, }
\end{aligned}
$$

for every real numbers $u, v, \alpha_{i}, \beta_{i}(i=0,1, \ldots, m)$.

The following Proposition is rather obvious. So the detailed proof of it is omitted.

Proposition 10 . Let

$$
\varphi(u, v)=\sum_{i=1}^{n} \theta_{i}\left|\zeta_{i} u-\rho_{i} v\right|
$$

where $\theta_{i}, \zeta_{i}, \rho_{i}$ are real number. If $\varphi\left(\rho_{i}, \zeta_{i}\right) \geq 0$ for all $i=1,2, \ldots, n$, then $\varphi(u, v) \geq 0$ for all $u, v$. 
Now, the above $L$ is of the form

$$
L=L(u, v)=\sum_{i=0}^{m}\left|\alpha_{i} v-\beta_{i} u\right| .
$$

By Proposition 10, $L(u, v) \geq 0$ for all $u, v$, if we prove that $L\left(\alpha_{i}, \beta_{i}\right) \geq 0$, $(i=0,1, \ldots, m)$.

Now, $L\left(\alpha_{i}, \beta_{i}\right)=0 \quad(i=1,2, \ldots, n)$ is obvious. So, it remains to prove that $L\left(\alpha_{0}, \beta_{0}\right) \geq 0$, i.e.

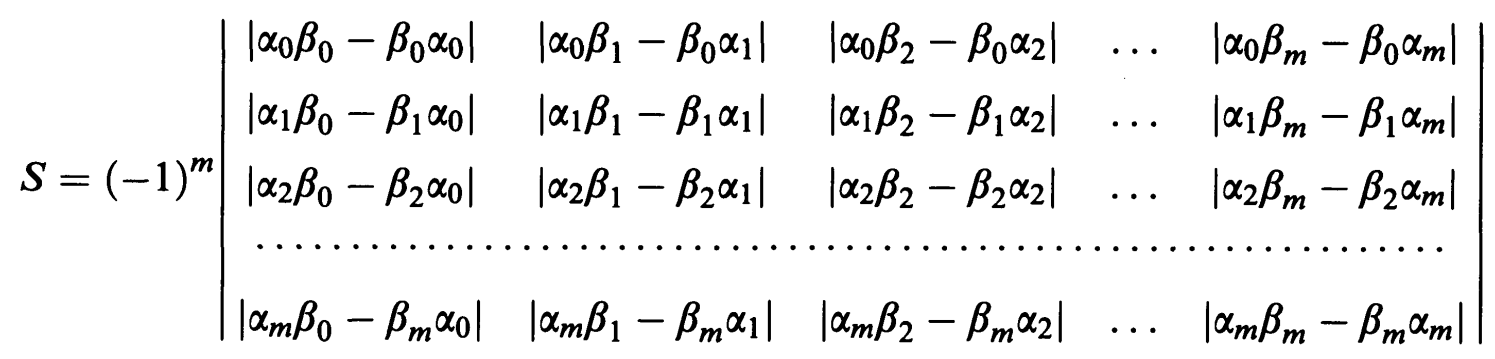

$\geq 0$.

Because of symmetry, we can assume that

$$
\frac{\alpha_{0}}{\beta_{0}} \geq \frac{\alpha_{1}}{\beta_{1}} \geq \frac{\alpha_{2}}{\beta_{2}} \geq \cdots \geq \frac{\alpha_{m}}{\beta_{m}}
$$

where $\beta_{i} \geq 0(i=0,1, \ldots, m)$ and if $\beta_{i}=0$ then $\alpha_{i}>0$ and $\alpha_{i} / \beta_{i}$ is regarded as $+\infty$. (If necessary, $\left(\alpha_{i}, \beta_{i}\right)$ is replaced by $\left(-\alpha_{i},-\beta_{i}\right)$ ). Then, $\left|\alpha_{i} \beta_{j}-\beta_{i} \alpha_{j}\right|=$ $\alpha_{i} \beta_{j}-\beta_{i} \alpha_{j}$, if $i \leq j$. Hence

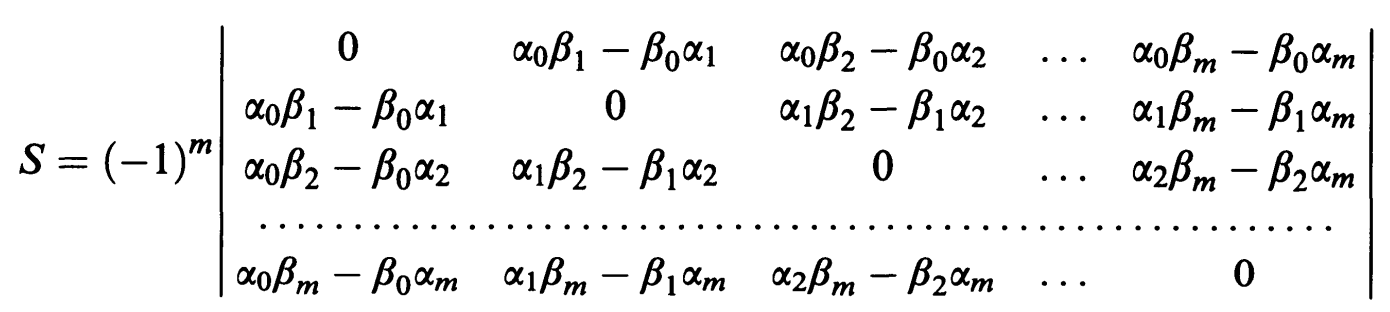

So, $S \geq 0$ follows from the following.

Proposition 11.

$$
S=2^{m-1}\left(\alpha_{0} \beta_{1}-\beta_{0} \alpha_{1}\right)\left(\alpha_{1} \beta_{2}-\beta_{1} \alpha_{2}\right) \cdots\left(\alpha_{m-1} \beta_{m}-\beta_{m-1} \alpha_{m}\right)\left(\alpha_{0} \beta_{m}-\beta_{0} \alpha_{m}\right) .
$$

Proof. $S$ is a homogeneous polynomial in $\left(\alpha_{i}, \beta_{i}\right)(i=0,1, \ldots, m)$ and vanishes when $\alpha_{i}=\alpha_{i+1}, \beta_{i}=\beta_{i+1}$. Also, it vanishes when $\alpha_{0}=\alpha_{m}, \beta_{0}=\beta_{m}$. Hence $S$ can be divided by

$$
\left(\alpha_{0} \beta_{1}-\beta_{0} \alpha_{1}\right)\left(\alpha_{1} \beta_{2}-\beta_{1} \alpha_{2}\right) \cdots\left(\alpha_{m-1} \beta_{m}-\beta_{m-1} \alpha_{m}\right)\left(\alpha_{0} \beta_{m}-\beta_{0} \alpha_{m}\right) .
$$


So,

$$
S=k\left(\alpha_{0} \beta_{1}-\beta_{0} \alpha_{1}\right)\left(\alpha_{1} \beta_{2}-\beta_{1} \alpha_{2}\right) \cdots\left(\alpha_{m-1} \beta_{m}-\beta_{m-1} \alpha_{m}\right)\left(\alpha_{0} \beta_{m}-\beta_{0} \alpha_{m}\right),
$$

for some constant $k$. In order to determine $k$, let $\alpha_{i}=m-i, \beta_{i}=1$ $(i=0,1, \ldots, m)$. Then, $\alpha_{i} \beta_{j}-\beta_{i} \alpha_{j}=j-i$. So,

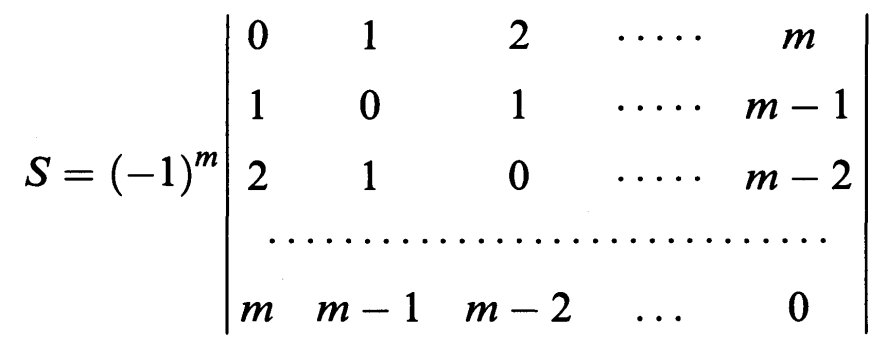

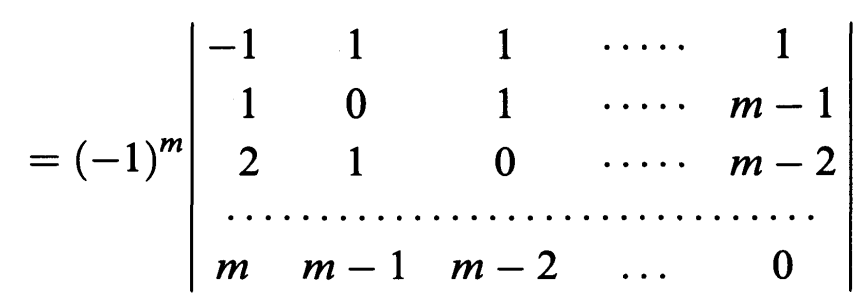

$$
\begin{aligned}
& =(-1)^{m}\left|\begin{array}{rrrrc}
-1 & 1 & 1 & \ldots \ldots & 1 \\
-1 & -1 & 1 & \ldots \ldots & 1 \\
2 & 1 & 0 & \ldots \ldots & m-2 \\
\ldots \ldots & \ldots \ldots \ldots \ldots \ldots \ldots \ldots & \ldots \ldots \\
m & m-1 & m-2 & \ldots & 0
\end{array}\right|
\end{aligned}
$$$$
=(-1)^{m}\left|\begin{array}{rrrrrrr}
-1 & 1 & 1 & 1 & \ldots \ldots & 1 & 1 \\
-1 & -1 & 1 & 1 & \ldots \ldots & 1 & 1 \\
-1 & -1 & -1 & 1 & \ldots \ldots & 1 & 1 \\
\ldots \ldots & \ldots \ldots & \ldots \ldots & \ldots \ldots \ldots & \ldots & \ldots \\
-1 & -1 & -1 & -1 & \ldots \ldots & -1 & 1 \\
m & m-1 & m-2 & m-3 & \ldots \ldots & 1 & 0
\end{array}\right|
$$

$$
=(-1)^{m}\left|\begin{array}{rrrrrrr}
0 & 2 & 0 & 0 & \ldots \ldots & 0 & 0 \\
-1 & -1 & 1 & 1 & \ldots \ldots & 1 & 1 \\
-1 & -1 & -1 & 1 & \ldots \ldots & 1 & 1 \\
\ldots \ldots \ldots \ldots \ldots \ldots \ldots \ldots \ldots \ldots \ldots . & \ldots \ldots \ldots & \ldots \\
-1 & -1 & -1 & -1 & \ldots \ldots & -1 & 1 \\
m & m-1 & m-2 & m-3 & \ldots \ldots & 1 & 0
\end{array}\right|
$$




$$
=(-1)^{m}\left|\begin{array}{ccccccc}
0 & 2 & 0 & 0 & \ldots \ldots & 0 & 0 \\
0 & 0 & 2 & 0 & \ldots \ldots & 0 & 0 \\
-1 & -1 & -1 & 1 & \ldots \ldots & 1 & 1 \\
\ldots \ldots \ldots \ldots & \ldots \ldots & \ldots & \ldots \ldots & \ldots & \\
-1 & -1 & -1 & -1 & \ldots \ldots & -1 & 1 \\
m & m-1 & m-2 & m-3 & \ldots \ldots & 1 & 0
\end{array}\right|
$$

$$
\begin{aligned}
& =(-1)^{m}\left|\begin{array}{ccccccc}
0 & 2 & 0 & 0 & \ldots \ldots & 0 & 0 \\
0 & 0 & 2 & 0 & \ldots \ldots & 0 & 0 \\
0 & 0 & 0 & 2 & \ldots \ldots & 0 & 0 \\
\ldots \ldots & \ldots \ldots & \ldots \ldots & \ldots \ldots \ldots \ldots & \ldots & \\
0 & 0 & 0 & 0 & \ldots \ldots & 2 & 0 \\
-1 & -1 & -1 & -1 & \ldots \ldots & -1 & 1 \\
m & m-1 & m-2 & m-3 & \ldots \ldots & 1 & 0
\end{array}\right| \\
& =2^{m-1} m \text {. }
\end{aligned}
$$

Hence,

$$
2^{m-1} m=k \cdot 1 \cdot 1 \cdots 1 \cdot m \text {. }
$$

Hence $k=2^{m-1}$, as was to be proved.

This completes the proof of Theorem 1. 